The Introduction of Transcatheter Aortic Valve Implantation (TAVI) Clinical, patient experience, economical and occupational hazard issues

\title{
Gry Dahle
}

\author{
The Intervention Center \\ and \\ Department of Cardiothoracic Surgery, \\ Oslo University Hospital, Rikshospitalet
}

Oslo Norway

Institute of Clinical Medicine

Faculty of Medicine

University of Oslo 
(C) Gry Dahle, 2020

\section{Series of dissertations submitted to the} Faculty of Medicine, University of Oslo

\section{ISBN 978-82-8377-656-0}

All rights reserved. No part of this publication may be reproduced or transmitted, in any form or by any means, without permission.

Cover: Hanne Baadsgaard Utigard.

Print production: Reprosentralen, University of Oslo. 


\section{ACKNOWLEDGEMENTS}

The present study was conducted at The Intervention Centre and Department of Cardiothoracic Surgery, Rikshospitalet, Oslo University Hospital during the years 2009-2014. The project was supported by a grant from The South-Eastern Regional Health Authority of Norway.

The path to fulfill this work has sometimes been rather tortuous and not always straightforward. Without the support from my main supervisor Professor Erik Fosse, this thesis would not have been completed. With his patience, encourage, engagement, knowledge and passion regarding new techniques and quality of life, I was guided through all these years. I also express enormous gratitude to my co-supervisor and colleague Kjell Arne Rein, without him standing by all the procedures and support in clinical day I could not have made this. His knowledge in the field has been invaluable and his support helped me maintaining progress in the project. I have to thank my second co-supervisor Professor Arnt Fiane for his support and providing me the opportunity to conduct this research in a busy surgical department.

A great thank to Jacob Bergsland for the initiation of this study.

I will also thank Vinod Mishra for supporting me with the economic research and good discussions, as well as Karl Øyri for audiovisual support.

Jo Eidet has been very supportive and helpful in data interpretation and statistics in addition to the collaboration in the study and the writing.

I thank Anette Aarsnes and Anne Catrine T. Martinsen from Institute of Physics for collaboration and co-writing in the hazard of irradiation.

Great thanks also go to the staff at the Intervention Centre and to our scrub nurses who were very helpful throughout all the implantations. The same gratitude I want to express to Per Steinar Halvorsen, Andreas Espinoza, Jan Hovdenes, the nurses at the Dept. of Anesthesia for the support in the hybrid operation room as well as the interventional cardiologists standing by during the procedures .The echo cardiologists, especially Jon Offstad, have been of great support in the pre-, per- and postoperative evaluation of the patients. 
Finally, I have to express gratitude to my friends and family who have mentally supported me throughout the whole period of this work. Especially, I am grateful to my generous mother who has been a continuous motivator and to her own concerns I have not always been very empathetic and patient during these years. 


\section{LIST OF CONTENTS}

\section{ACKNOWLEDGEMENTS}

SUMMARY OF THE THESIS

LIST OF PAPERS

\section{INTRODUCTION}

\section{AORTIC STENOSIS}

- History of aortic stenosis

- Epidemiology and etiology

- Clinical presentation and prognosis

- Diagnosis and evaluation

\section{SURGICAL TREATMENT OF AORTIC VALVE STENOSIS}

- Open heart-traditional surgery

- Suture-less- and rapid deployment valves, further development of biological valves

- Valvular sizing for open heart surgery

TRANSCATHETER AORTIC VALVE IMPLANTATION-TAVI

- History of transcatheter aortic valve implantation -TAVI

- Access routes in TAVI

- Hazard of imaging/radiation

- Hybrid operation room

\section{COST, QUALITY OF LIFE AND VALUE-BASED MEDICINE}

- Cost and reimbursement

- Quality of life (QoL)

- Value-based medicine

- Value-based medicine and introduction of new technology 


\section{AIMS OF THE STUDY}

- The aims

- The hypothesis

\section{MATERIAL}

- Study design and population

- Implementation and eligibility

\section{METHODS}

- Investigation in general

- TAVI procedure

- Quality of life assessment

- Specific investigations for the different papers

1. Impact of the introduction of TAVI on costs, clinical outcome, hospital stay and quality of life

2. Safety and efficiency of alternative access routes and devices. Development of new technology

3. Relation between intraoperative improvement of the left ventricular function to long term morbidity, mortality, functional capacity and quality of life

4. Radiation burden on the patient and staff involved in the procedure

\section{SUMMARY OF RESULTS}

- Clinical outcome and quality of life

- Specific for each paper

1. Impact of introducing TAVI on costs, clinical outcome, hospital stay and quality of life

2. Safety and efficiency of alternative access routes and devices. Development of new technology

3. Relation between intraoperative improvement of the left ventricular function to long term morbidity, mortality, functional capacity and quality of life

4. Radiation burden on the patient and staff involved in the procedure

\section{DISCUSSION}

General discussion

- Specific for each paper

1. Impact of introducing TAVI on costs, clinical outcome, hospital stay and quality of life

2. Safety and efficiency of alternative access routes and devices for TAVI. Development of new techniques

3. Relation between intraoperative improvement of the left ventricular function to long term morbidity, mortality, functional capacity and quality of life

4. Radiation burden on the patient and staff involved in the procedure 
TAVI MANAGEMENT FOLLOWING THIS STUDY

- Expanding the indications

ORGANIZATION AND TECHNOLOGY FOR MINI INVASIVE TREATMENT OF STRUCTURAL HEART DISEASE

LIMITATIONS

CONCLUSIONS

REFERENCES 



\section{LIST OF PAPERS}

This Thesis is based on the following publications which will be referred by Roman numerals.

I. Dahle G, Rein KA, Fiane A, Fosse E, Khushi I, Hagen T, Mishra V. Innovative technology transcatheter aortic valve implantation: Cost and reimbursement issues. Scand Cardiovasc J 2012; 46: 345-352.

II. Dahle G, Rein KA. Direct Aorta Ascending Approach in Transcatheter Aortic Valve Implantation. Innovation 2014; 9:1-9.

III. Eidet J, Dahle G, Bugge JF, Bendz B, Rein KA, Aaberge L, Offstad JT, Fosse E, Aakhus S, Halvorsen PS. Long-term outcomes after transcatheter aortic valve implantation: the impact of intraoperative tissue Doppler echocardiography. ICVTS 2016; 23:403-409.

IV. Aarsnes A, Dahle G, Fosse E, Rein KA, Aaberge L, Martinsen AC. Evaluation of occupational radiation dose in transcatheter aortic valve implantation. Radiation Protection Dosimetry. 2018; $179(1): 9-17$. 



\section{SUMMARY}

Innovation is a natural process in the evolution of health service to the society. It involves many stakeholders such as the patients, the physicians, the payers, the politicians, the engineers, the regulators, the scientific societies etc. (1).

When introducing a new treatment option, it is of great importance to study the value of the new therapy both in a patient as well as a societal perspective at an early stage as possible. Thus, this study aimed at investigating and elucidating the cost of the new TAVI treatment, the objective results in heart function and complications, the quality of life for the patients and the hazards for the personnel in the hybrid operation room.

The idea of value-based medicine is to record the treatments effect on the health outcome that matter for the patient and to relate this improvement to costs (2). Objective measurements on health improvements in cardiac patients are in this study defined as quality of life and New York Heart Association (NYHA) functional class. When introducing TAVI in our institution, a prospective study was designed with the approval from the ethical committee. Costs, changes in quality of life and clinical outcomes were recorded from the first day, and a clear picture of the potential value outcomes was obtained. As treatment initially was offered to patients with advanced disease, a number of patients were observed who did not benefit from the treatment.

A better selection is mandatory in order to save patients from a non-beneficial treatment and the burden of an unnecessary intervention. Our findings indicate that it may be possible to improve the selection, but further studies are warranted.

For the first patients receiving a TAVI procedure the costs were higher than the Diagnosis-related Group (DRG) reimbursement offered. The device cost was the main driver for cost counting for more than $50 \%$ and remained almost unchanged during the study period. TAVI may have a great impact on hospital budget in the future, but if the value for the patient increases, the innovation may be justified. 



\section{AORTIC STENOSIS}

\section{History of aortic stenosis}

The French physician Lanzare Rivière (1589-1655) provided what is probably the first description of aortic stenosis (3). He described a patient with progressively worsened condition of "occluded artery", shortness of breath and the disappearing of peripheral pulse before the patient died. At necropsy the left ventricle was enlarged and round masses occluded the "mouth" of the aorta, other necropsy reports followed (4).

One of the first persons to discuss the etiology of aortic stenosis was Jean Baptiste Bouillaud that in 1835 described a 37-year old man who had fever, acute arthritis and endocarditis and died (3). Bouillard found aortic stenosis at necropsy and believed the stenosis to be caused by endocarditis described as inflammation of the internal membranes of the heart and valves. The English physician Carl Edward Hasse challenged in 1846 Bouillard's theory by stating that "ossification cannot invariably be ascribed to endocarditis" and William Osler in 1908 mentioned the sclerotic and endocarditic causes of aortic stenosis. In 1947 a major study of 200 necropsies published by Thomas Karsner and Simon Koletsksy championed rheumatic cause of aortic stenosis (Calcific disease of the aortic valve. Philadelphia. JB Lippincott 1947).

Congenital cause of aortic stenosis was largely ignored by the previously mentioned investigators until Smith and Mattews quoted Paget who in 1844 discussed the bicuspid aortic valve(5). Later several physicians recognized the tendency of the congenitally bicuspid aortic valve to become stenotic.

Although the debate on cause continued it was generally agreed that calcific aortic stenosis was more common in males than in females. Based on 106 cases of autopsies published 1954 the ratio male: female was 3:1(6). 


\section{Epidemiology and etiology}

Aortic valve stenosis is the most frequently acquired heart valve disease in the Western world and after coronary artery bypass grafting (CABG), aortic valve replacement (AVR) is the second most common cardiac operation performed. The prevalence increases with age, at $50-59$ years it is $0.2 \%$ and in patents older than 75 years about $5 \%(7,8)$. Although degenerative aortic stenosis is most common, there are evidence of regional clustering and observations of family aggregation, suggesting that genetic components also contribute to the overall prevalence (9).

Calcific aortic stenosis was for a long time thought to be a consequence of a "passive degenerative process" driven by calcific deposition. Later research has demonstrated that the complex active process involving inflammation, lead to calcification and thickening of the leaflets.

In the Western countries, bicuspid aortic valve is the most common congenital heart defect with prevalence of $0.5-2 \%(10)$ and bicuspid aortic stenosis develops one or two decades earlier in these patients (11). Recent studies in China have demonstrated that up to $50 \%$ of the patients referred for treatment of aortic stenosis have bicuspid valve $(12,13)$. Rheumatic heart valve disease can also cause aortic stenosis, but is rare in the Western world (14).

Bicuspid aortic valves are classified as Sievers class 0 with no raphe: "true bicuspid" and Sievers class 1 and 2 with one or two raphes. The most common is Class 1 with raphe between the two coronary cusps $(15,16)$.

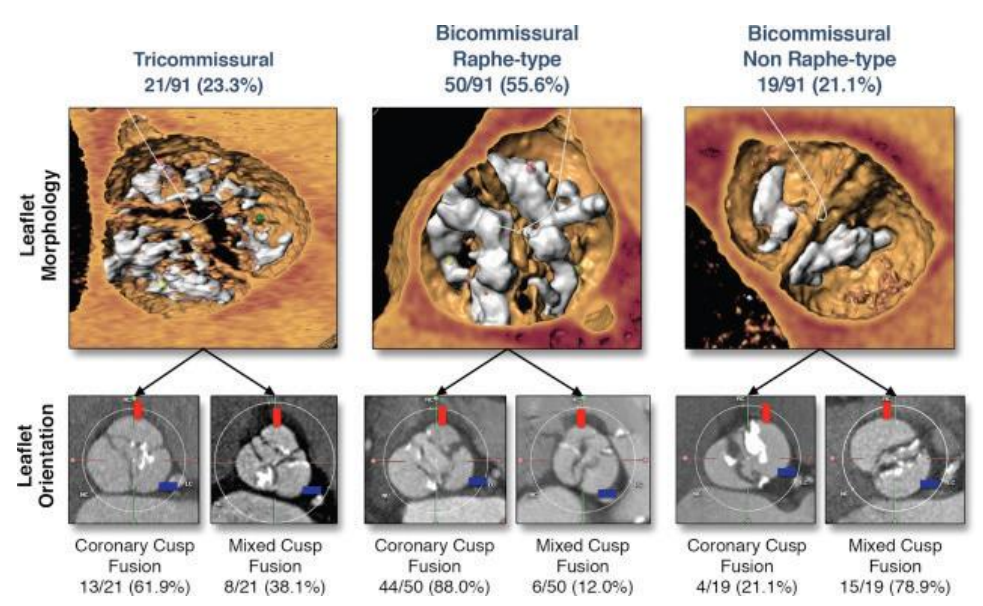

Figure 1. Classification of bicuspid aortic valve (15). 


\section{Clinical presentation and prognosis}

The spontaneous prognosis was according to William Stokes (1854) variable and dependent on 1) the severity of the obstruction 2) the degree of cardiomegaly 3 ) the regularity and intensity of the heart beat and 4) the duration of symptoms (3).

Asymptomatic aortic stenosis is associated with relatively low mortality (17). Untreated, $50 \%$ of patients with symptomatic, severe aortic stenosis will die within 2 years (18). Typical symptoms are dyspnea, angina and syncope. Dyspnea may be caused by the combination of impaired cardiac output and pulmonary arterial hypertension, due to increased filling pressure of the left ventricle and high left atrial pressure. Angina is experienced by about $35 \%$ of the patients. The mechanism may be increased oxygen demand due to muscle hypertrophy and decreased coronary flow reserve. Syncope during exercise is the most serious prognostic symptom. Due to reduced opening area of the aortic valve and exercise induced arrhythmias, the patient does not have the ability to increase cardiac output during exercise. A history of one or more syncope is a strong indication for urgent surgery.

\section{Diagnosis and evaluation}

\section{Auscultation}

Rene J.H. Bertin perceived a murmur coincident with ventricular systole in a patient with aortic stenosis. A more detailed description was provided by James Hope in 1832: “One (murmur) is heard during the ventricular contraction (i.e. with the first sound) on the sternum opposite the lower margin of the third rib, and thence for about two inches or more upwards, along the course of the ascending aorta towards the right; and it is louder in these situations than below the level of the valves. Its pitch or key is usually hat of a whispered $r$....."(3). 


\section{Catheterization and angiography}

Cardiac catheterization was introduced in 1929. Today all the patients with suspected aortic stenosis are catheterized to evaluate the coronary arteries and measure pressure gradient over the aortic valve (3).

\section{Echocardiography}

Transthoracic and transesophageal echocardiography have improved the diagnosis of aortic stenosis. Left ventricular function status, concomitant valve disease, myocardial thickness, pressure gradient and area of the aortic valve, mono/bi/ tricuspid valve, annular dimensions are all factors to categorize any data to evaluate the severity of aortic stenosis as mild, moderate or severe (19). Stress echocardiography is sometimes necessary to demonstrate the gradient, especially in low flow low gradient stenosis and paradoxical aortic stenosis.

\section{SURGICAL TREATMENT OF AORTIC VALVE STENOSIS}

\section{Open heart - traditional surgery}

Already in 1902 Lauder Brunton proposed surgical intervention in patients with valvular stenosis. The idea was considered radical at that time and first in $1913 \mathrm{~T}$. Tuffier performed the first operation for aortic stenosis by digital dilatation (3)104/106. Surgical efforts to treat valvular stenosis in the 1920s were directed toward the mitral valve by Elliott Carr Cutler and Samuel Levine by their cardiovalvulotome, but 10 of the 12 treated patients died because of mitral regurgitation and congestive heart failure, and the operation was stopped in 1929 (20). In 1950 an aortic valve commissurotomy was performed and described by R.P Glover et al with a dilation instrument from the left ventricle(21). Charles Hufnagel et al implanted in 1952 a caged ball valve in the descending aorta in a patient with aortic regurgitation(22). 
Further development of Hufnagel's operation was done by H.Swan and A.B. Kortz in 1955 when first performing a direct vision operation on the aortic valve, an ice bath was used to induce hypothermia and the aortic valve was widened by commissurotomy (23).

Shortly after the first successful surgeries with extra-corporal circulation in 1957(24), several centres started exploring the possibility to replace the aortic valve (25).

One of the first mechanical aortic valves for replacement was the Starr-Edwards (Albert Starr and Lowel Edwards) cage-ball valve, developed for use in 1960 (26), first implanted in Norway by Leif Efskind 1964 (27). The concern with the Starr-Edwards valve was fracture of the cage and thrombus formation (28).

The next generation valves consisted of a lid held in place by two braces, a tilting disc, most common the Swedish Björk-Shiley, first implanted in 1971 (29). The Norwegian Karl Victor Hall together with the American engineer Bob Kaster developed in 1977 the Medtronic-Hall valve (Medtronic Inc., Minneapolis, MN) with the same tilting disc concept(30).

Ten years later these valves were gradually replaced by a valve type consisting of two lids hinged in the middle, the bi-leaflet valves. First in use was the St Jude (St Jude Medical Inc., St. Paul, MN) introduced in 1977. The valve is robust and still in use, proven to be very durable with good hemodynamics (25).

The first biological valves became available in 1965 but showed poor durability. Durability improved when Alain Carpentier introduced the use of glutaraldehyde for fixation $(31,32)$. The leaflets were initially mounted on a delrin frame, later the so-called stent less valves without the frame were developed. Still the biological valves are less durable than the mechanical, but the advantage is that the patients do not have to be on life-long systemic anticoagulation.

The trend in AVR has been to move from mechanical to biological valves $(33,34)$. 


\section{Suture-less - and rapid deployment valves, further development of biological}

\section{valves}

All the surgical valves required excision of the existing valves and fixation of the artificial valves by sutures. To reduce time on the heart-lung-machine, experiments on suture-less valves were started. In 2008 experience with the 3F ATS Enable (Medtronic, Minneapolis, MN), no longer in use, was reported(35). Then the first studies on Perceval (Liva Nova, London, UK) came in $2009(36,37)$ and later the Intuity (Edwards Lifesciences, Irvine, CA) a rapid deployment valve came up as an alternative (38). The advantages of these were shorter time on cardiopulmonary bypass and easy implantation via mini sternotomy. However, the rapid deployment- as well as the suture-less valves seemed to be more associated with paravalvular leak than the sutured valves, and of atrioventricular block requiring pacemaker. In addition, they were more expensive than surgical valves $(39,40)$.

\section{Valvular sizing for open heart surgery}

Comparison of hemodynamics between the different biological valves is complex as valve sizers, the valves themselves, manufacturers`sizing and suggested sizing strategies are inconsistent. Duenst et al (41) concluded that the sizing and implantation technique has much greater impact on postoperative valve hemodynamics than valve brand or type, "Initially one has to avoid patient prosthesis mismatch". In the era of TAVI and valve-in-valve procedures, it is also becoming important to "prepare for a later valve-in-valve". There is ongoing work to standardize valvular sizing, a collaborative effort between the European Association for Cardiothoracic Surgery (EACTS), the American Association of Thoracic surgery (AATS), and the Society of Thoracic surgeons (STS). This effort aims at identifying the most important fields in intraoperative prosthetic heart valve sizing and labelling where future standardization is necessary (42). 


\section{TRANSCATHETER AORTIC VALVE IMPLANTATION-TAVI}

\section{History of Transcatheter Aortic Valve Implantation -TAVI}

The suture-less surgical valves paved the way for catheter-based insertion of valves, and already in the 1980s a research group at Skeiby hospital in Århus developed a valve that could be inserted via a catheter. Thus, the Danish cardiologist Henning Ruud Andersen performed the first aortic valve implantation in 1989 in the descending aorta of a pig that survived the procedure. This technique was many years later the start of transcatheter aortic valve implantation (TAVI) when Henning Ruud Andersen implanted a "homemade" stent valve in the descending aorta (19).

However, the response in the cardiac surgical community was limited, and a small American company bought the patent for 10,000 USD. In 2002 Alain Cribier did the first-in-man TAVI with the Cribier-Edwards valve, and thereafter the technique has evolved (43). The indications have expanded, new valves and delivery systems have been developed as well as different access routes for placing the valve. As TAVI was a completely new technique with no documentation of the longterm performance of the valve, the procedure was first recommended to patients considered otherwise inoperable. As the experience grew, indications have been widened, and today TAVI seems like an acceptable alternative to open surgery in many patients.

\section{Access routes in TAVI}

In the early era of TAVI, the dimensions of the delivery catheters were large and not fit for small femoral arteries. Caution was also made to severe calcification, tortuous vessels and aneurysms with thrombus formation. Thus, alternative accesses like transapical direct aortic and via the subclavian artery were explored. The access was chosen based on CT (computer tomography) reconstruction (44). Only the apical access is antegrade through the valve. The access site is chosen based on valve pathology and anatomical features. 
Transapical approach was preferred in redo situations with vein grafts originating from ascending aorta and with calcification in this part of aorta. In this situation the pericardium is often adherent and thickened. When there is low ejection fraction, thin ventricular wall with aneurysm and fragile, "soft myocardium" after prednisolone treatment and pulmonary disease, the direct aortic approach is preferred. As the dimensions of the delivery catheter became smaller and the experience increased, most TAVIs today are performed transfemorally with preclosure systems in local anesthesia and conscious sedation. New alternative access routes have also been added like transaxillary, transcarotid, transcaval and transjugular.

\section{Hazards of imaging/radiation}

During cardiac interventions, both patient and operators are exposed to radiation. For the patient, this is most often a one-time event with little health consequences, while for the operators this is an occupational hazard. The occupational dose obtained during cardiac interventions may vary largely, depending on equipment, protection and procedure (45).

$\mathrm{Hp}(10)$ is the recommended dose quantity for assessment of effective dose, assuming uniform whole body exposure and $\mathrm{Hp}(0.07)$ to measure eye lens dose. Effective $(\mathrm{E})$ is a quantity defined by the International Commission on Radiological Protection (IRCP) and is a weighted sum of several organ doses. The algorithm from ICRP Publication 120 for estimation based on single dosimeter reading outside the led apron was used:

$\mathrm{E}=\mathrm{Hn} / \sqrt{ } 21$

$\mathrm{Hn}$ is the $\mathrm{Hp}(10)$ dose measured unshielded in the neck height(46).

Personal dosimeters are provided and mandatory to use in Norway. The dose limitation per year at Oslo University Hospital is; Effective dose whole body: 20mSv, Equivalent dose Eye lens: $20 \mathrm{mSv}$ and Equivalent skin dose: $500 \mathrm{mSv}$. 
In the era of expanding the use of cardiac interventions, there are several preventive measures: the use of lead coats, moveable protective shields, lead glasses, pre procedural planning to minimize radiation time, focus on fluoroscopy pulse rate and training of all involved personnel (47). Especially the eye lens dose has been focused regarding cataract development. Surgeons often have the hands in the radiation field and no good protection gloves are available. In addition, the surgeons need to be positioned near the patient and the radiation field. In transfemoral procedures the operators may stand with some distance to the fluoroscopy field. New imaging modalities may in the future replace angiography $(48,49)$.

\section{Hybrid operation room}

A hybrid operation room is a surgical theatre that is equipped with advanced medical imaging devices such as fixed C-Arms, CT scanners or magnetic resonance imaging (MRI) scanners. A hybrid suite allows combined open surgical and interventional procedures. It may be used for mini-invasive surgical procedures or interventional cardiological procedures. A hybrid suite facilitates conversion from closed interventions to open surgical procedures when needed, as the room is fully equipped with cardiac surgical tools including a heart-lung machine (50).

\section{COST, QUALITY OF LIFE AND VALUE-BASED MEDICINE}

\section{Cost and reimbursement}

In Norway, the actual diagnosis-related group (DRG) code is automatically created by a combination of International Classification of Diseases (ICD)-diagnostic code and procedure code based on the Nordic Classification of Surgical Procedures (NOMESCO) from 2010. Main- and secondary diagnostic system algorithms allocate specific DRG to each patient. When TAVI was introduced in Norway in 2008 , there was no DRG reimbursement, nor any procedural code. Before establishing a procedural 
code for TAVI, the Conformité Européenne (CE) approval in Europe and the Food and Drug Administration (FDA) approval in US had to be obtained. For the Edwards Sapien ${ }^{\mathrm{TM}}$ valve the first CE approval was given in 2007 (FDA 2011). In 2011 the Sapien XT valve had CE mark (FDA 2014) and in the end Sapien 3 valve had CE mark in 2014 with FDA approval in 2015. The FDA approval was slightly behind the European approval for any version of the Sapien ${ }^{\mathrm{TM}}$ valve. The competitor, the selfexpandable Medtronic CoreValve ${ }^{\mathrm{TM}}$ had first CE mark in 2010.

Then a procedural code and subsequently a DRG reimbursement formula could be made. The reimbursement for TAVI differs between countries, thus the penetrance of TAVI varies country wise. Germany is on the top with highest reimbursement value (51) and highest rate of TAVI vs Surgical aortic valve replacement (SAVR). In Norway the first dedicated DRG for TAVI was set in 2012.

\section{Transcatheter AVR - Clinical Data Sources}

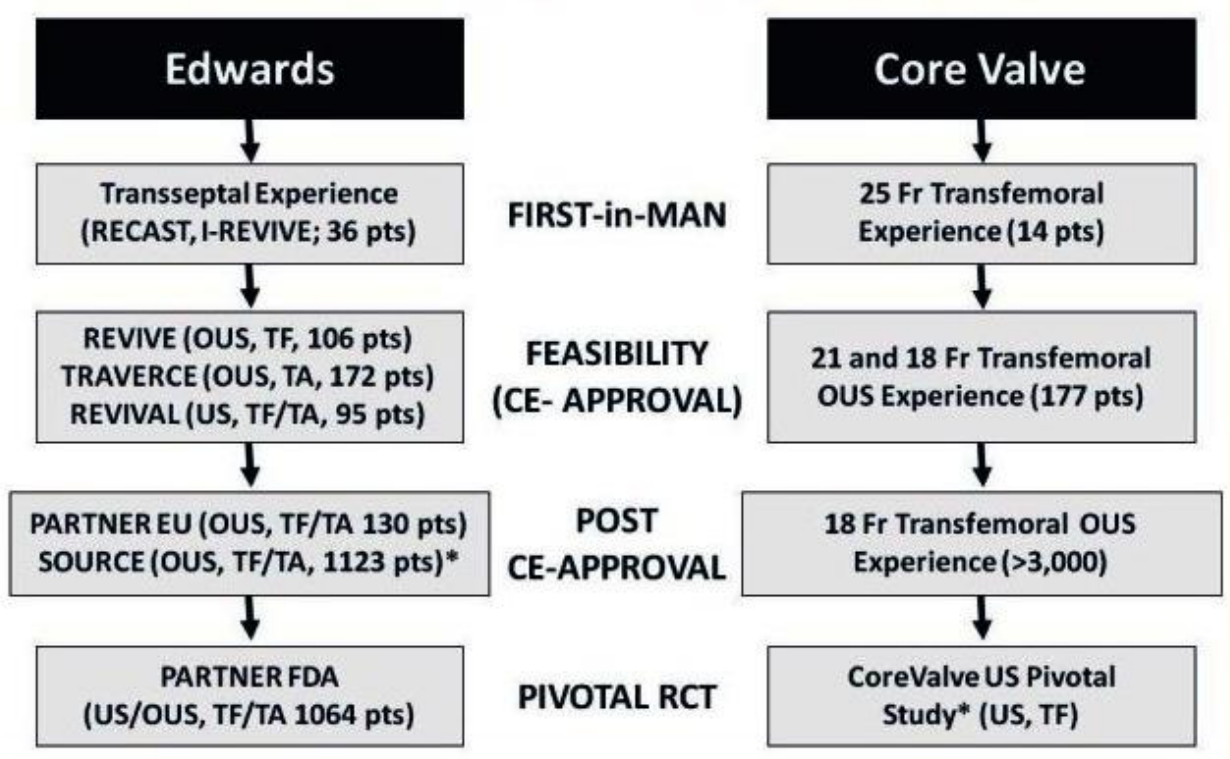

Figure 2. The pathway to CE approval for Edwards Sapien ${ }^{\mathrm{TM}}$ and Medtronic CoreValve ${ }^{\mathrm{TM}}$ (52). Through different studies the new devices have to prove their safety and efficiency. 


\section{Quality of life, QoL}

Quality of life $(Q \circ L)$ is the general well-being of individuals, outlining negative and positive features of life. It observes satisfaction, including all from physical health, family, education, employment, wealth, finance and safety. Different measurement tools exist. In health care the Medical Outcome Study Short Form 36 version 2 (SF 36V2) is commonly used (53). The SF-36 is a 36 -item patient reported survey of patient health used to compare the situation before and after an intervention. The patients self-complete the questionnaires and according to a scoring system a final estimate of quality of life is determined.

Especially in the introduction of new devices, the quality of life scoring has been used to evaluate the effect on the patients in addition to the economics and survival. Combining the quality of life, survival and economics, the value of care (V) of the procedure can be calculated, Figure 3. A new study reveals that despite of better psychological outcomes among TAVI treated patients, $25 \%$ develop anxiety or depression, and the mortality in such individuals is higher than in non-depressed patients (54).

\section{Value-based medicine}

The term value-based health care was first introduced by the economist Michael Porter in 2010 (55). He defined value as the outcomes that matter to the patients related to the cost of delivering the outcomes, i.e. health outcomes achieved per dollar.

Value of care is an evolving concept in which medical reimbursements will be based on outcomes divided by the cost of care delivered. "Perfect care" can be defined as an expedited and sustainable patient recovery with no impairment of health status after minimal health care cost spent $(55,56)$. I.e., value means efficiency and value-based health care has been argued for as a superior framework for performance improvement in health care. Cardiothoracic surgeons have tradition to report outcomes to prove good standards across specialty and should be ready to deliver value. 


\section{Principles of Value-based Health Care Delivery}

Value $=\frac{\text { Health outcomes that matter to patients }}{\text { Cost of delivering the outcomes }}$

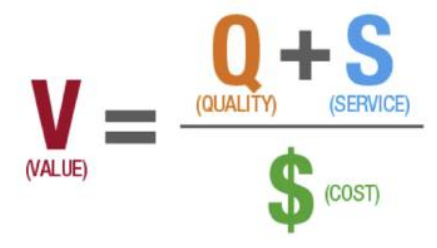

Value is measured for the care of the a patients medical condition over the full cycle of care

- Outcomes are the full set of health results for a patient's conditions over the care cycle

- Costs are the total costs of care for a patient's condition over the care cycle

Figure 3. The value calculation, Porter ME 2010 (55). Porter set up a formula to calculate the value of treatment $(V)$ for the patient. It will be the health outcomes that matters for the patient $(Q+S)$ divided by the cost (\$) of delivering these outcomes.

\section{Value-based medicine and introduction of new technology}

Value-based medicine is a healthcare delivery model in which providers, including hospitals and physicians, are paid based on health outcomes $(55,56)$. Under value-based care agreements, providers are rewarded for helping patients improve their health, reduce the effects and incidence of chronic disease, and live healthier lives in evidence based way. This contrasts fee-for-service or capitated approach, in which the providers are paid based on the amount of healthcare service they deliver. In Norway the DRG system is based on this model.

When introducing a new model, a fee-for-service approach seldom gives good outcome. Thus, when introducing new methods like transcatheter aortic valve implantation we applied a value-based framework for study outcome, investigating the direct cost, the subjective and the objective outcomes for the patients, the hazard for the personnel and the safety of new access. 


\section{AIMS OF THE STUDY}

\section{The aims}

The aim of this thesis was to investigate and characterize the challenges of implementing new technology in a surgical program in a value based framework, where cost, patient safety and quality of life and the environmental hazard for the personnel were considered along with technical issues and clinical outcome.

The main aims of the Thesis were:

1. To investigate the impact of the introduction of TAVI on costs, clinical outcome, length of hospital stay and the patient's quality of life.

2. To describe the changes in quality of life after TAVI treatment (data are not published).

3. To evaluate the safety and efficiency of alternative access routes and devices. Development of new techniques

4. To describe the relationship between intra-operative improvements of the left ventricular function to long term mortality, morbidity, functional capacity and quality of life (QoL).

5. To describe radiation burden on the patient and the staff involved in the TAVI procedure.

\section{The hypothesis}

1. The costs would increase with the use of new expensive devices, though the reduction of hospital stay would compensate for some of the extra cost of the device. The quality of life would improve after TAVI treatment. Together this may increase the value for the patient.

2. The clinical effect of the treatment on the patients and quality of life would correlate with improved left ventricular function.

3. Radiation dose would be a limiting factor for the staff in the operating room and might be harmful to the patient. 


\section{MATERIAL}

Already at start of the TAVI program, all patients eligible for this new procedure were included in a study protocol following informed consent. Quality of life questionnaires were completed, and echocardiography and clinical evaluations performed according to a specifically designed protocol both pre- and postoperatively for all patients, Figure 4.

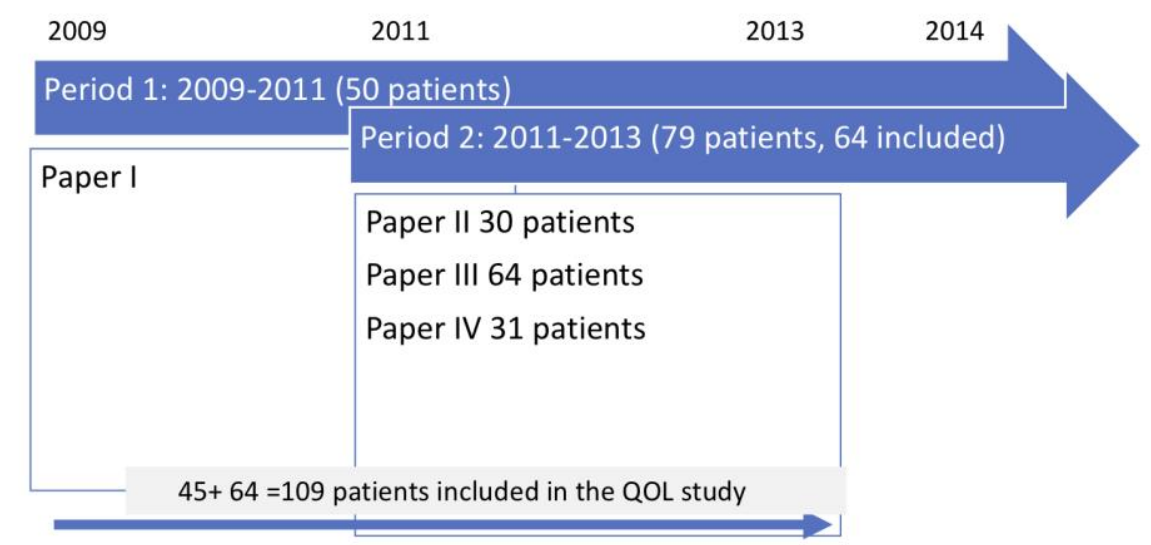

Figure 4. In Period 1 the first 50 patients were included in Paper I. In Period 2 the next 79 patients were included, 15 were excluded for the Paper III study. From this population 30 patients were included in the Paper II and 31 patients were included in Paper IV. From Period 1: 45 and from Period 2: 64 patients were included in the total quality of life assessment.

\section{Study design and population}

1. To study the cost of TAVI procedures a retrospective, single centre, cohort study was performed of the costs of the consecutive 50 very first TAVI patients treated at Rikshospitalet, from October 2009 to September 2011.

2. In order to study the changes in quality of life after TAVI, a prospective single centre cohort study was performed. The self-reporting SF36v2 questionnaire was completed for the 109 
included patients at admission and at one-year follow up. These data are not published in any separate paper.

3. To demonstrate the feasibility of direct aortic access a prospective, single centre study was performed in the first 30 consecutive patients treated with this technique at Rikshospitalet, Oslo University Hospital (OUS) from October 2011 to June 2013.

4. In order to study the selection of patients for TAVI and the possible benefit for the patient's experience we performed a prospective, single centre cohort study. The quality of life and corresponding echocardiographic findings and NYHA classifications in 64 consecutive patients treated with TAVI from September 2011 to June 2013 with one year follow up, both femoral and central access, were recorded.

5. To estimate the radiation health hazards in TAVI, we performed a prospective, single centre study of the difference in radiation dose exposure for the operators completing either transfemoral or trans-apical TAVI in 31 consecutive patients treated 2012 to 2013.

\section{Implementation and eligibility}

1. All patients treated with TAVI from the start in Sept. 2009 to June 2013 were asked for informed consent to collect data on patient characteristics, echo findings, complications and quality of life at one year follow up. The patients were asked consecutively, and all agreed. Retrospectively the costs were recorded in Paper I for the first 50 patients. In this group the first five patients missed the inclusion to the quality of life evaluation due to technical aspects with the questionnaire analysis. In the whole study, 109 consecutive patients completed a SF36 form at admission and at one-year follow up.

2. When starting with direct aorta TAVI, the first 30 patients treated with either Edwards Sapien $\mathrm{XT}^{\mathrm{TM}}$ valve (Edwards Lifesciences, Irvine, $\mathrm{CA}$ ) or CoreValve ${ }^{\mathrm{TM}}$ (Medtronic, Minneapolis, MN) via mini sternotomy or mini thoracotomy were included, described in Paper II.

3. In Paper III, 64 consecutive patients treated with TAVI from Sept 2011 to June 2013 were included, some of these were also included in the direct aorta access cohort. Fifteen patients 
were excluded: four due to treatment using local anesthesia, which made transesophageal echocardiography inconvenient, eight due to poor image quality, three due to logistic issues disturbing the procedures (television broadcasting companies in operation room).

4. In Paper IV the radiation dose of the implanters was prospectively collected for 15 direct transaortic approach treated patients and 16 patients with transfemoral approach during the years 2012-2013.

\section{METHODS}

\section{Investigations in general}

All patients were discussed in a multidisciplinary team of surgeons, interventional cardiologists and echo cardiologists. The patients had preclinical assessment with clinical history including NYHA class, coronary angiography, echocardiography, CT scan and quality of life assessment. The indication for intervention was decided and the procedural access discussed. All patients signed an informed consent approved by the ethical committee. The value of the treatment that matters for the patient could then be determined from the improvement in NYHA classification and quality of life divided by the costs of delivering these outcomes.

\section{TAVI procedure}

The first 25 patients were all treated with access from apex, subsequently changing to transfemoral TAVI and direct aortic approach was the last introduced. Different prototypes of valves were used, they were both balloon expandable and self-expandable valves, Figure 5. 


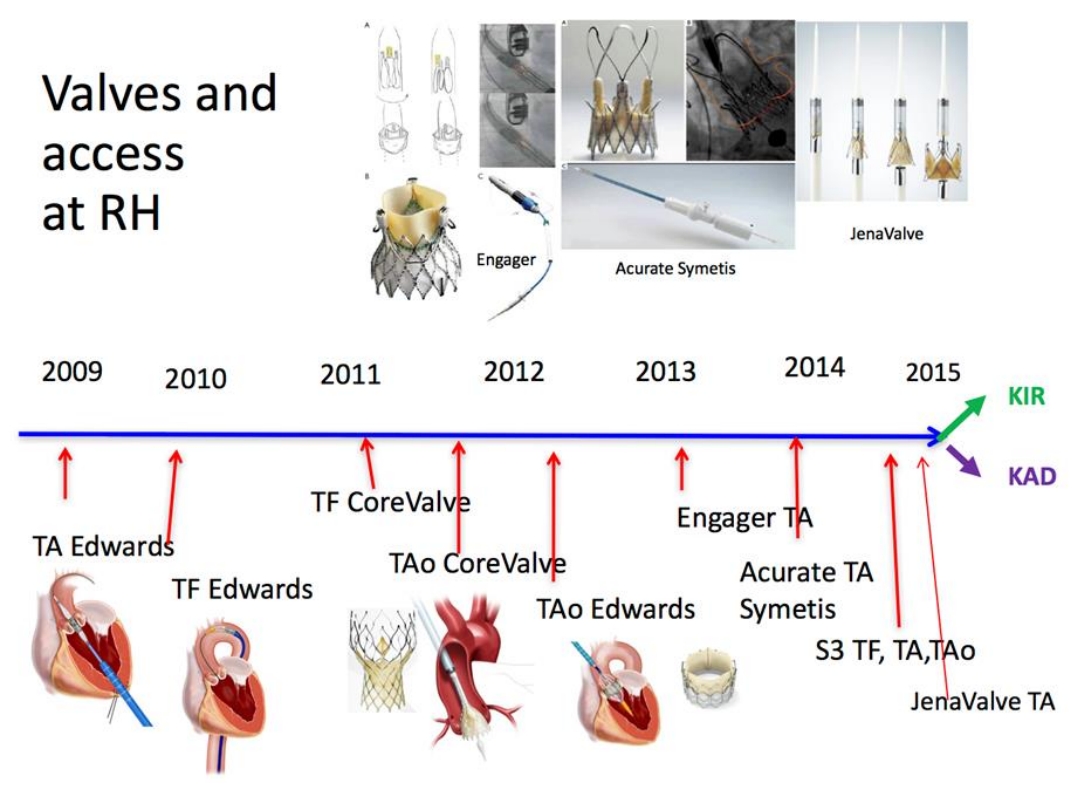

Figure 5. The time frame of implementation of the different valve types and access routes at Rikshospitalet until the split in pathways in 2015. The patients then became tracked through Department of Cardiology (KAD) for femoral access and the Department of Cardiothoracic Surgery for central access (KIR).

All patients were treated in a hybrid operation room, with transesophageal echo and angiography guidance in general anesthesia.

In order to evaluate cardiac function, all patients were examined by echocardiography pre-, per- and postoperatively, before discharge, at 3, 6 and 12 months.

\section{Quality of life assessment}

In order to assess the quality of life, the medical outcome study short form 36 version 2 (SF 36v2) was used. Each patient had to make a self-assessment at admission and at 12 months follow up. The SF 36 questionnaire is based on a scoring system addressing eight dimensions of health characterizing both physical- and mental capacity of the patient. It consists of eight scaled scores from 0-100 on: vitality, physical functioning, bodily pain, general health perceptions and physical role functioning, emotional role functioning, social role functioning and mental health. In addition, 
the total Physical and Mental scores may be calculated as the ninth and tenth scales, which are the summary of both the physical and mental components four in each group, by using specific software (Quality Metric Inc. Optum, Lincoln, RI, USA). A higher score reflects a better perception of quality of life.

The patients were gathered into three groups (Figure 4):

- First group (Period 1) was the first 50 patients treated, described in cost analysis, clinical outcomes including NYHA classification and quality of life (45 patients).

- Second group (Period 2) was the next 64 patients included, described in clinical outcomes, NYHA class, echo findings and quality of life.

- Third group (all patients in group $1+2$ ) describing quality of life assessment.

p-values were calculated using the SPSS V18 (SPSS Inc., IBM, Chicago, IL, USA).

\section{Specific investigations for each paper}

1. Impact of the introduction of TAVI on costs, clinical outcome, hospital stay and quality of life

Clinical outcomes such as morbidity and mortality were recorded, and the hospital costs were calculated for the first fifty patients treated by TAVI. All patients filled in a SF 36 short form to record quality of life, but these data were not published in paper I. As this study was performed in the first patients eligible for TAVI, $50 \%$ of the patients underwent a transapical procedure. Decision was made to first use only one TAVI system, the Edwards Sapien /Sapien XT ${ }^{\mathrm{TM}}$ balloon expandable valve, and 42 procedures were completed, 25 with transapical and 17 with transfemoral delivery. Additionally, 
eight patients were treated with the CoreValve ${ }^{\mathrm{TM}}$ system, as it was designed for treatment of bigger annuli.

The cost analysis involved two sets of data, one based on data for the individual patient (direct costs) and one based on the overhead costs (indirect costs), with the overhead costs ultimately also allocated to the individual patient. All cost data were calculated in 2010 -prices and the basic principle of the analysis were to record as much as possible of the resources used as direct cost to the individual patient $(57,58)$. The motivation for this study was the lack of DRG reimbursement for TAVI procedures in Norway, and the need of creating such a financing platform. Scientific figures of costs were urgently needed.

The demography as well as peri- and postoperative clinical records were made in addition to in hospital complications and death. The Medical Study Short Form 36 version 2 (SF 36v2) was used to assess each self-reported quality of life at the preoperative assessment and at the 12 months follow up. The patients were divided into two groups with either a transapical or a transfemoral access. In this first period, the learning curve was extremely steep. As the first 25 patients treated had a transapical access and the next 25 patients had a transfemoral approach, there may be a selection bias regarding results. The length of stay (LOS) and time in the intensive care unit were also recorded and used in the economic analysis.

\section{Safety and efficiency of alternative access routes and devices. Development of new} technology

The direct ascending aorta approach was introduced to be the second central approach becoming an alternative for patients with poor ventricular function. As a quality control, the first 30 patients treated with direct aortic access were recorded in a feasibility study. No comparison was done between the two valve systems or between mini sternotomy versus mini thoracotomy. The demography, morbidity, mortality, procedural success and complications were recorded. 
The patients were evaluated by the "Heart Team", accepted for TAVI, and if not suitable for femoral access because of anatomical issues, allocated for either transapical or direct aortic access.

Depending on the position of the ascending aorta, the relation to the sternum, the brachiocephalic vein, any possible vein grafts, left and right internal mammary artery graft from previous surgery, either right mini thoracotomy or mini sternotomy was selected. Reconstruction of CT scan was done to decide access and to perform sizing for the transcatheter valve from perimeter, diagonal length measurements and surface area calculations. According to the size and shape of the annulus, calcium distribution, the length of the ascending aorta and the ventricular function (pacing tolerance) the most appropriate valve system was chosen.

The room setup in a direct ascending aorta procedure was different from transapical and transfemoral set up with the two cardiac surgeons at the head of the patient and the anesthesiologist at the feet.

\section{Relation between intra operative improvement of the left ventricular function to long term morbidity, mortality, functional capacity and quality of life}

The first 50 patients were treated until August 2011. During the next period from September 2011 to June 2013,64 consecutive patients were included prospectively.

The patients were divided into "Responders" and "Non-responders" based on echo findings. The predefined cut-off value of $>20 \%$ average increase in the longitudinal peak systolic velocity was defined as responder and below non-responder.

The aim was to identify the patients who would benefit from TAVI treatment and identify the risk factors for cardiac death. The two groups were compared with respect to cardiac death, improvement of quality of life three to twelve months after treatment, and the risk of cardiac events. A standard 2D transthoracic echocardiogram was obtained using the Vivid E9 ultrasound scanner from GE (Vingmed, Horten, Norway). The Medical Outcome Study Short-Form 36 Version 2 (SF-36v2) was used to assess each self-reported quality of life before and after the TAVI procedure at 3 month 
and 1 year, higher the score, the higher the perception of quality of life. The dimensions were grouped in two categories: the physical component score and the mental component score. The TAVI procedures were performed in the hybrid operation room under general anesthesia with echo and fluoroscopy guidance with Edwards Sapien ${ }^{\mathrm{TM}}$ and CoreValve ${ }^{\mathrm{TM}}$ implanted either transfemorally with "cut down" or transapical / transaortic. The patients came for follow up with clinical examination, transthoracic echocardiogram, NYHA class evaluation and quality of life questionnaire.

\section{Radiation burden on the patient and staff involved in the procedure}

In order to evaluate the radiation risk to the staff, dosimeters (one for body and one for eye lens) were applied for the operators for 31 consecutive patients in the same period as the enrolment of intraoperative improvement of ventricular function. The dosimeters of the operators were analyzed for the occupational dose gained for each procedure. Total patient dose area product (DAP) was calculated, which means the DAP acquired during fluoroscopy and image acquisition. The position of the operators and the access route used were recorded.

Two cardiothoracic surgeons and one cardiologist were using Electronic personal dosimeters EPD MK2+ to measure the personal dose equivalent $\mathrm{Hp}(10)$ for the body (at the breast height outside led apron) and Unifors EDD-30 electronic dosimeters to measure $\mathrm{Hp}(0.07)$ for the eye lens (sensors on the left side of the glasses).

The patient radiation doses were based on the radiation dose descriptor dose-area product (DAP) provided by the $x$-ray unit. It was noted where in the operating room the different operators were standing and which role each of them had in the procedure. 


\section{SUMMARY OF RESULTS}

\section{Clinical outcomes and quality of life}

There was a significant difference between Period 1 and 2 with respect to the need of chest compression and use of heart lung machine during and after valve implantation. No significant difference was recorded between the two periods with respect to Logistic EuroScore, the use of aortic balloon pump and one year mortality, the NYHA classification for baseline and one year follow up were also similar, Table 1, Figure 6.

\begin{tabular}{|c|c|c|c|}
\hline Period & 1 & 2 & $\mathrm{p}$-value \\
\hline Years & Sept 2009-Aug 2011 & Sept 2011- June 2013 & NS \\
\hline Central/TF & $25 \mathrm{TA} / 25$ & $36 * / 28$ & $\mathrm{NS}$ \\
\hline Logistic EuroScore & 32.5 & 29.4 & $\mathrm{NS}$ \\
\hline $\begin{array}{c}\text { Chest compression } \\
\text { during procedure }\end{array}$ & $11(22 \%)$ & 0 & $\mathrm{p}<0.001$ \\
\hline HLM & $5(10 \%)$ & 0 & $\mathrm{p}<0.009$ \\
\hline IABP & $4(8 \%)$ & $2(3.1 \%)$ & $\mathrm{NS}$ \\
\hline New PM & $1(2 \%)$ & $12(18.8 \%)$ & $\mathrm{p}<0.005$ \\
\hline NYHA classification baseline & $50(100 \%)$ in class 3-4 & $56(88 \%)$ in class 3-4 & NS \\
\hline NYHA classification 1 year & $34(68 \%)$ in class 1-2 & $39(61 \%)$ in class $1-2$ & NS \\
\hline Mortality 1 year & $11(22 \%)$ & $13(20.3 \%)$ & NS \\
\hline
\end{tabular}

Table 1. A comparison of EuroScore, peri- and post procedural incidents and morbidity between the first 50 patients treated (Period 1) and the following 64 patients (Period 2).

* 12 TA and 24 Tao.

HLM: heart-lung machine, IABP: intra-aortic balloon pump, TA: transapical, Tao: Transaortic, TF: transfemoral. 


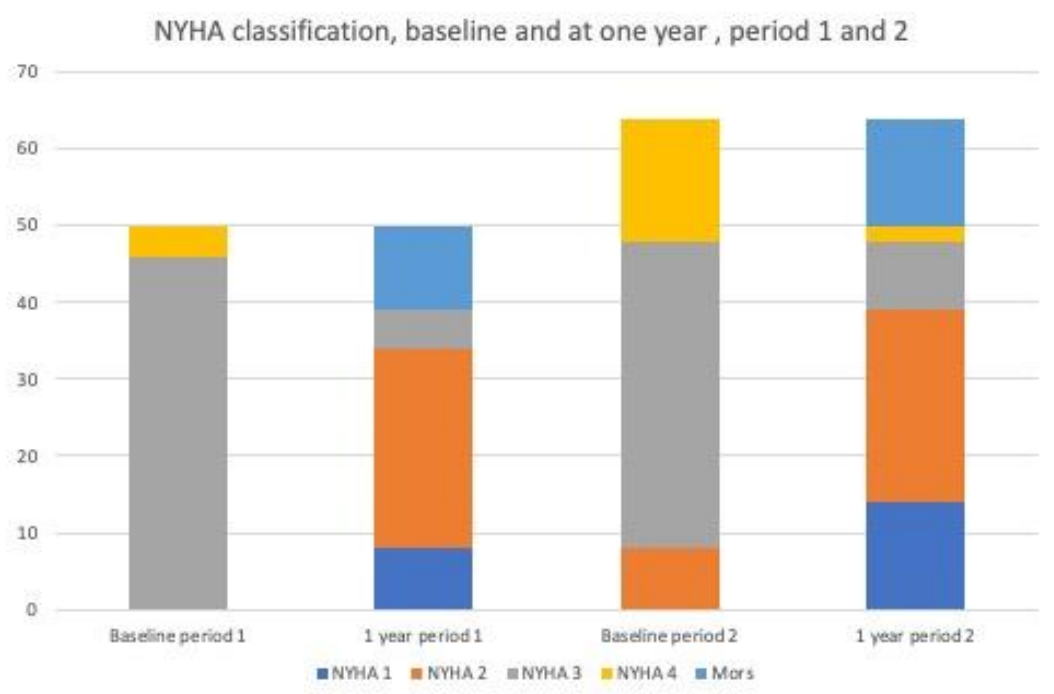

Figure 6. A summary of the changes in NYHA classification at baseline and at one year follow up for the two periods.

In the total population, a paired sample test was performed for all modalities of the four physical and mental scorings of the SF 36 form. A significant improvement was found in all modalities except from the Bodily pain with a $p$ value of 0.082 . However, there were some drop outs due to mortality and patients who did not come for the follow up. Some patients did not reply to all the questions (range 45-32 \%) in the SF 36 questionnaire.

For Period 1 there were no significant change in Bodily Pain, General Health and Role Emotional, around $66 \%$ completed the form. For Period 2 the change in Bodily Pain, General Health, Mental Health and Physical Component Score were not significant, in this group only $60 \%$ of the patients completed the form. For the total group of patients the change in Bodily Pain was the only nonsignificant parameter, Table 2 and Figure 7. 


\begin{tabular}{|c|c|c|c|c|c|c|c|c|c|}
\hline \multirow{2}{*}{$\begin{array}{l}\text { Difference in score } \\
\text { Baseline - } 1 \text { year }\end{array}$} & \multicolumn{3}{|c|}{ Group 1 (45 patients included) } & \multicolumn{3}{|c|}{ Group 2 (64 patients included) } & \multicolumn{3}{|c|}{ Group 3 (109 patients) } \\
\hline & $\mathrm{n}$ & value & $p$ & $\mathrm{n}$ & value & $\mathrm{p}$ & $\mathrm{n}$ & value & $\mathrm{p}$ \\
\hline Physical function, PF & 31 & 10,78 & 0,024 & 41 & 15,52 & 0,001 & 72 & 13,49 & 0,000 \\
\hline Role-physical,RP & 29 & 17,81 & 0,004 & 36 & 13,19 & 0,061 & 65 & 15,26 & 0,001 \\
\hline Bodily pain, BP & 31 & 6,36 & 0,110 & 36 & 6,09 & 0,292 & 67 & 6,21 & 0,082 \\
\hline General health, GH & 31 & 4,91 & 0,262 & 43 & 6,38 & 0,107 & 74 & 5,76 & 0,048 \\
\hline Vitality, VT & 31 & 15,32 & 0,001 & 41 & 7,16 & 0,043 & 72 & 10,67 & 0,000 \\
\hline Social function, SF & 31 & 14,11 & 0,023 & 39 & 12,50 & 0,032 & 70 & 13,21 & 0,002 \\
\hline Role emotional, RE & 26 & 9,61 & 0,430 & 34 & 25,49 & 0,003 & 60 & 18,60 & 0,009 \\
\hline Mental health, $\mathrm{MH}$ & 31 & 8,36 & 0,017 & 41 & 2,68 & 0,274 & 72 & 5,12 & 0,012 \\
\hline Physical component score, PCS & 29 & 3,98 & 0,035 & 35 & 3,56 & 0,070 & 64 & 3,75 & 0,006 \\
\hline Mental component score, MCS & 29 & 5,13 & 0,044 & 35 & 3,90 & 0,023 & 64 & 4,45 & 0,002 \\
\hline
\end{tabular}

Table 2. A summary of the results for each subgroup of quality of life. The value is indicating the difference in score between baseline and at one year follow up.

$\mathrm{n}$ : number of patients completing the questionnaire.

\section{Quality of life,} differences in scoring, baseline and one year follow up

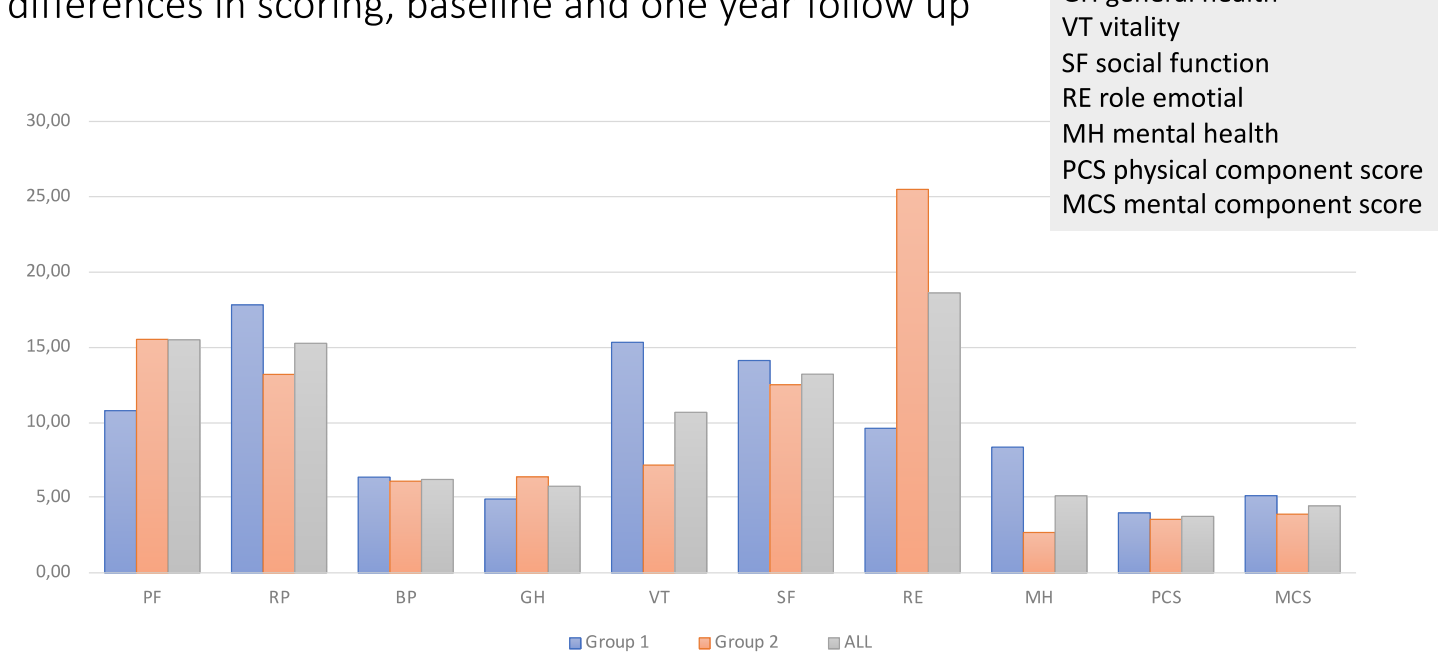

PF physical function RP role-physical BP bodily pain GH general health VT vitality SF social function $\mathrm{RE}$ role emotial $\mathrm{MH}$ mental health PCS physical component score MCS mental component score

Figure 7. The Quality of life for the different parameters in each period: Period 1 (45 patients included), Period 2 (64 patients included) and Period 3 all patients included (109). 


\section{Specific for each paper}

1. Impact of introducing TAVI on costs, clinical outcome, length of hospital stay and the patient's quality of life

The very first 50 patients treated with TAVI in our centre were included.

There were 31 males aged mean 82 (59-92) years, the mean Logistic Euro Score was 33\% (range 774), the mean ejection fraction was $44 \%(20-55)$ and all the patients were in NYHA class III or IV. Altogether 42 procedures were performed with the Edwards Sapien ${ }^{\mathrm{TM}}$ platform, 25 transapically and 17 transfemorally. In addition, eight transfemoral procedures were performed with the CoreValve ${ }^{\mathrm{TM}}$ Revalving system.

Only one new pacemaker was implanted post procedurally. Seven (14\%) patients died within 30 days and the one year mortality was 11 patients (22\%). Eleven patients (22\%) needed external chest compression, five patients (10\%) were on partial bypass and four patients $(8 \%)$ needed postoperative intra-aortic balloon pump. Together with the logistic Euro Score of 33\%, this reflects the high-risk population that was included in the introduction of the new method. The 30-day mortality for all the patents was $14 \%$, but for the first 25 patients the 30 -day mortality was $24 \%$.

This influenced the costs, as the main difference in cost was due to the length of stay.

There were need of mechanical support (external chest compression, partial bypass and IABP) in half of the patients, 13 (52\%) for the first 25 patients and 20 (40\%) for all.

In the first 25 patients the length of stay was mean 199 hours (range 77-362), while in the overall material this was mean 185 hours (range 49-362).

For the actual (total) costs a mean reduction of 120000 NOK (20000 US\$) was calculated during the inclusion period. The estimate from the trend variable indicated that total costs were reduced by 1650 NOK (275 US\$) per patient added.

The device cost was the main cost driver with the price of 172500 NOK (28750 US\$). The actual costs and the current DRG reimbursement were totally different from each other, with a much 
higher actual cost. The latter was mean 312522 NOK (52087 US\$) for transfemoral TAVI and 334140 NOK (55690 US\$) for transapical TAVI, while the reimbursement was only 237330 NOK (39555 US\$). The procedural time was almost equal for transfemoral and transapical access.

During the study period a reduction in time consumption was observed. The early mortality and complication rate were higher in the first 25 patients than in the second half of the patients, all of whom were treated by transapical access. The subsequent patients were treated with transfemoral access if anatomy allowed for it.

There was a variation in the length of stay, mean $185 \mathrm{~h}(49-362 \mathrm{~h})$ with a mean cost of $55,537 \$$. The length of stay before procedure and the total length of stay also showed a great variation, some of it dependent of which day the patients were treated. The stay in ICU was the most homogenous one. There was also a correlation between the number of procedures performed and cost, with a slight decline in costs with the increasing numbers of procedures performed.

Disaggregating total costs into different cost drivers revealed that $52 \%$ of the costs for the transapical TAVI were related to device vs $55 \%$ for transfemoral. The length of stay was contributing to $34 \%$ of the total costs for the patients treated with transapical access while it counted for $33 \%$ of the costs for the patients treated transfemorally. This difference was not significant.

\section{Safety and efficiency of alternative access routes and devices for TAVI. Development of new techniques}

Thirty patients were included in the first study to explore the direct aortic approach.

The mean age was 80 years and $60 \%$ were men with mean logistic Euro Score $33 \%$ and most in NYHA

Class 3-4. More than half of the patients had coronary artery disease and $20 \%$ abdominal aortic aneurysm, reflecting the vascular comorbidities.

In 29 of the 30 patients the valve was successfully implanted (97\%), one patient underwent periprocedural valve-in-valve implantation because of initial high deployment of the first valve. A mini-sternotomy was used in 21 patients. The largest valve size was dominating, median size 29 $\mathrm{mm}$ (range 23-31) $\mathrm{mm}$. One patient had a repair of ventricular perforation on cardiopulmonary 
bypass, no other serious complications were observed. Twenty two patients were extubated in the operation room. There were six patients with paravalvular leak > grade 3 . Five patients got a permanent pacemaker. There were no cerebrovascular events, coronary obstructions or annulus rupture. The mean procedural time and time spent in operation room were 137 (93-466) and 250 (114 -551) minutes. Two patients died within 30 days and total survival was $87 \%$ (observation time 118 months).

3. Relation between intra-operative improvement of the left ventricular function to long term mortality, morbidity, functional capacity and quality of life

Retrospectively 64 patients, 35 males, mean age 82 years (73-86) were included.

Thirty-five patients (55\%) were classified as responders and 29 patients $(45 \%)$ were classified as nonresponders.

The inclusion period was from September 2011 to August 2013. For the total group the NYHA class was 3-4 in 56 patients (88\%), and mean logistic Euro Score was $26.7 \%$ (15.6-43.6), higher for the nonresponders, 29.8 (18.1-43.0). No circulatory support was needed for any of the patients.

The requirement of permanent pacemaker was similar in the responder and non-responder groups after the procedure, $34 \%$ vs $31 \%$.

On periprocedural transesophageal echocardiography the peak systolic velocity used as a measure of left ventricular longitudinal function before and after valve implantation increased on average $20 \%$ or $0.9 \mathrm{~cm} / \mathrm{s}$ in the responder group, whereas it remained unchanged among the non-responders. The responder and the non-responder group had significant and similar reduction in mean pressure gradients across the aortic valve at 12 months follow up, whereas the ejection fraction was almost unchanged (a slight reduction in the non-responders, with mean EF down from $50 \%$ to $47 \%$ ). Non-responders had three-fold higher risks of death compared to the responders during the 12 months follow up. Twenty-eight of the included 64 patients exhibited one or more adverse cardiac event, 19 of the 29 in the non-responder group and 9 of the 35 in the responder group. Significant long-term improvements in NT-proBNP and left ventricular function were observed only in 
responders. The SF 36 scores were improved in all categories for the responders. This correlates well to the NYHA class that was markedly improved in the responders, almost $50 \%$ in NYHA class I and $40 \%$ in NYHA class II at one year follow up. For the non-responders there was only slightly improvement in NYHA class and none improved to class I. There were only slight improvement in SF 36 scores and deterioration in the score for Social Function and General Health for the nonresponders. Moreover, the cardiac death and total mortality were significantly higher in the nonresponder versus the responder group.

\section{Radiation burden on the patient and the staff involved in the procedure}

A total number of 31 procedures were included, some enrolled during the same period as for the direct aorta study. The radiation exposure to the patient and operator were compared between patients receiving transfemoral and transaortic procedures with a mean age of s 81.5 vs 84 years for the two groups. The body mass index (BMI) was slightly higher in the transfemoral group. The median total patient dose area product -value was $75.9 \mathrm{~Gy} \mathrm{~cm}^{2}$ in transaortic and $130 \mathrm{~Gy} \mathrm{~cm}^{2}$ for transfemoral access. In the transaortic access group, a significant difference in body doses outside the led apron and equivalent doses to eye lenses were detected for the main surgeon (CTS1), compared to the other surgeon (CTS2) and the cardiologist (C).

The median fluoroscopy time was the same in both groups (16 minutes). Linear regression analysis indicated a stronger correlation for surgeons between patient body mass index (BMI) and fluoroscopy DAP in transaortic than in transfemoral access. 


\section{DISCUSSION}

\section{General discussion}

All studies included in this Thesis describe different aspects of implementing new technology such as

TAVI as a treatment option for patients with aortic stenosis at Oslo University Hospital,

Rikshospitalet. From September 2009 patients who were otherwise inoperable were included. For

safety reasons the first 25 patients were all treated through a transapical access. The delivery

catheters were of large dimension, many patients had peripheral vascular disease and all were treated in general anesthesia. The procedure was performed in a hybrid operation room with back up of heart-lung machine. The patients were selected from an extremely high-risk cohort, and during the procedure some were in need of cardio-pulmonary bypass, intra-aortic balloon pump and external chest compression. The mortality was high throughout the study period. For safety reasons, the patients stayed longer in the hospital than strictly necessary. The economic burden for the hospital was high and the benefit for the patient quite uncertain with respect to this new treatment option. In the implementing phase a randomized approach was not realistic. But in order to monitor the economic impact on the hospital budget and the burden to the patients, an economical and a quality of life evaluation were implemented from the very first patient allocated to the TAVI treatment. This enabled a value based approach for evaluating the new method, and also provided precious information for a national DRG reimbursement rate according to the real costs.

Our material is unique as it includes the first treated patients from day one of the program. It is possible to observe a learning curve for the patient stay, treatment time and complications. Furthermore, right from the first patient treated, informed consent was obtained, and the patients were included in a database. With this tool we were able to study important value based factors for health care related to this TAVI technology. Sub-studies to evaluate specific issues in detail were performed, one to address new access route (Paper II), one to evaluate the hemodynamic response correlated to the quality of life and NYHA classification and one to focus on the occupational 
radiation for the operators (Paper IV). Thus, quite early a complete overview was obtained for many quality factors related to the new method.

It was of great importance to justify the value of the method demonstrating a high morbidity rate and a one year mortality of more than $20 \%$. The patients who survived had a significant improvement in quality of life and more than half of them ( $55 \%$ responders) obtained a better heart function. These findings were in support of the method, convincing the health authorities that it was safe to proceed with transcatheter aortic valve implantation, but the selection of patients had to be made better. For the complete group of patients all factors contributing to the quality of life score showed an improvement, except for Bodily Pain. The patients were frail and they had many reasons for having pain.

Elimination of the physical discomfort of having aortic stenosis was not enough to free them from other bodily pain. A way of identifying patients who would benefit from TAVI treatment could be the evaluation of the intraoperative ventricular function. Most importantly it was to define a method to identify the potential responders before the treatment was executed in order to have a therapy of the highest value both for the patient and the society.

Since the introduction of TAVI the risk of mortality score has been continuously declining into a lower risk population and currently one discusses how low the risk score should be defined for offering a TAVI procedure to any patient with aortic stenosis. The low risk patients may be treated in an easier and faster way without ICU service and general anesthesia. Recently FDA approved TAVI treatment in low risk patients with significant aorta stenosis based on two publications in New England Journal of Medicine from $2019(59,60)$. The total stay may be reduced and thereby the total costs for transcatheter valve treatment.

For the first patients receiving a TAVI valve the cost were higher than DRG reimbursement offered. The DRG reimbursement was 229660 NOK and the actual costs were mean 311847 NOK, ranging from 281604 to 361282 NOK. The device cost was the main driver for cost, counting for more than $50 \%$ and remained almost unchanged during the study period. 
For the first 45 patients (Period 1), there was a significant improvement in SF 36 regarding Physical Performance, Physical role, Vitality, Social Function, Mental Health and Summary of Physical and Mental Health. This reflects an acceptable value of treatment for the patients that survived. The burden of comorbidities and frailty was extremely high with the Logistic Euro Score of 33\%. Due to dropouts because the mortality was high (11 patients) the cohort became small. Nevertheless, there was a significant improvement for all modalities except from Bodily Pain, General Health, and Emotial Role. For the Physical Summary results and mental summary results the p-value was 0.035 and 0.044 .

For the next 64 patients (Period 2) there was a significant improvement of Physical Function and Emotional Role with $p$ values of 0.001 and 0.003 as was also the improvement for vitality, social function and mental component score $(p<0.023-0.043)$. If this group had been splitted into responders and non-responders even a tendency of decrease in score for general health and social function for the non-responders could have been observed, however, not significant. This may reflect the very frail population in this study. For the responders there were significant improvements in physical function, general health, social functional and role emotional, Figure 7.

There was no difference in survival between the two periods.

The two groups, Period 1 and Period 2, were not comparable, but the impression was that the patient selection, treatment performance and survival improved during the observation period and the value of the treatment for the patient was better for the second group of patients. For the whole period including all 109 patients, there was a significant total improvement for all factors in quality of life except from Bodily Pain, which was a consequence of high age and morbidity in the cohort. Taken into consideration that the non-responders had hardly any improvement, this was compensated for by all the responders who could take advantage of the treatment, contributing to a better quality of life after TAVI altogether for the whole group. 
In the future with artificial intelligence (AI) and machine learning different predictors for the benefit of any treatment, also for TAVI, may be identified and the "value of treatment" better evaluated (6163).

Even for the short time of evaluation from 2009 to 2013 the "value of treatment" was increased because of experience, better selection, and improved technology.

The real cost driver was still the TAVI device as the costs were not reduced. The length of stay as well as complications and morality were recorded. The quality of life improvement was calculated, and "the value of treatment" could be estimated as "the health outcome that matters for the patient" divided by "the cost of delivering outcomes". At present it may be possible for the hospital administration to "save money" on TAVI patients. Though a recent report from the Norwegian Institute of public Health: Health Technology Assessment, Part 2 Health economic evaluation performed cost-utility analysis of TAVI compared to surgery for intermediate risk patients The conclusion was a modest health gain (incremental effectiveness: 0.07 QALYs) at higher costs (incremental costs: 71000 NOK). The calculated incremental cost-effectiveness ratio was equal to 1.04 million NOK per QALY gained in the base case scenario. The calculated absolute shortfall for patients with severe aortic stenosis and the intermediate surgical risk receiving standard treatment was equal to 3.6 QALYs.

The inclusion period was in the early TAVI era 2009-2013 with a cohort of patients older than 80 years and Logistic EuroScore of $25-30 \%$. At present the patients appear to be younger with less comorbidity. It would have been of interest to perform a similar evaluation of the TAVI patients of today to see whether the difference between responders and non-responders still persists. Even though the question today is not which patients are too frail to benefit from TAVI treatment, but rather which patients are too healthy for transcatheter valve therapy! It would be an economical benefit and helpful for the patients to have some parameters to predict the advantage of TAVI. Unfortunately, we only have these per-operative parameters for assessment. 
It would be even more useful with preoperative factors predicting the benefit. Further investigation will be of importance and the machine learning technology may in this respect become useful (64). When moving into the era of low risk patients, it is important to focus on adverse events compared to the surgical aortic valve replacement. The issue of durability is yet not fully answered and will be of utmost importance when moving into the cohort of younger patients.

Our study of these very first patients treated with TAVI may be considered as a pioneer work with respect to value based medicine. Several modalities from clinical aspects, to economy and quality of life will contribute to the value of treatment for the individual patient. This ought to be done for all new treatment options when introduced into clinical practice to demonstrate the usefulness of the method, despite any potential hazards.

To make the TAVI procedure simplified at the same time as one move into less morbid and younger patients, may increase the procedural risk. If the procedure is removed from the hybrid operation room it may be even worse and eventually reduce possibility to save the patient if complication occurs.

The importance of a Heart Team performing the cases is crucial. The tendency today to create two separate pathways for transcatheter valve treatment, a parallel to what we have seen for many years with respect to the treatment of coronary artery disease is regrettable $(65,66)$.Insufficient collaboration between specialties may be harmful to the patient and does not comply with the concept of the highest value based medicine treatment. 


\section{Specific for each paper}

1. Impact of introducing TAVI on costs, clinical outcome, length of hospital stay and the patient's quality of life

As TAVI was a new treatment method for aortic stenosis, and the device costs were high, it became urgent to provide data on in-hospital costs when this technology was introduced in clinical practice. Our data were of great importance when later the level of DRG reimbursement was calculated for TAVI procedure in Norway.

No significant difference in patient characteristics for transapical and transfemoral access in the 50 first consecutive patients was found, though there were some differences in morbidity and mortality. Recent studies have shown more favorable results for the transfemoral patients (67). Some of these differences may be correlated to the learning curve for the procedures, as the program started with transapical access and then was switched to primarily transfemoral access. There was also a learning curve regarding selection of patients for the procedure, hence some of this knowledge could be beneficial when allocating the following 25 patients for transfemoral approach. In addition, the delivery catheters became smaller and the experience increased. Most TAVIs nowadays are performed transfemorally with preclosure systems in local anesthesia. New alternative access routes have also been explored such as transcarotid, transaxillary, transcaval and transjugular. All these factors may contribute to shorter stay and reduced costs.

The first patients in this program were extremely frail with several comorbidities. They were old, mean age over 80 years and at very high surgical risk. External chest compression was done in $22 \%$ of the procedures and post procedural intra-aortic balloon pump (IABP) was needed in $8 \%$ of the patients. This was reflected by a 30 -day mortality of $14 \%$ and a total mortality of $22 \%$ (observation time 4-26 months). This might have influenced the length of stay and thereby the costs. There was a significant discrepancy between actual hospital costs and the current Norwegian DRG 
reimbursement for TAVI procedure which was the same as for surgical aortic valve replacement settled in 2010. This discrepancy was mainly explained by excessive costs related to the cost of the device and the introduction of a new program with new technology. For safety reasons, extra precautions were taken and the patients had extended monitoring. Cost innovations should be considered in price-setting of reimbursement for novel technology. There was a slight decrease in costs as increasing number of procedures were performed. Though, by far the actual costs were covered by the reimbursement. The main reasons were: 1 ) the device cost 2) all procedures performed under general anesthesia 3) extended length of stay because of inexperience in own hospital and lack of competence in the referring hospitals and logistics regarding best week day of admission 4) increased need of man power 5) frail patients and complications.

This paper documented the real cost of a new procedure and the need of a special procedural code to cover this by DRG. This was in the early era of TAVI and experiences, imaging tools and better patient selection have reduced the procedural time, length of stay and mortality. The device costs however, are still approximately the same.

There are suggestions for extra DRG coding for "new technology add-on payment" (NTAP) in a recent publication in Guide point Reimbursement Resources (68), which may be of importance for implementation of new technology in the future.

It is worth noting that the device cost contributed to over $50 \%$ of the total costs both for TAVItransapical and TAVI-transfemoral access.

If the study had been performed today, the device costs would have contributed to most of the total costs, as the number of persons involved in the procedure would be less. Many of the patients do not have ICU stay and the total length of stay is minimized, "fast track" policy. A study from our centre has recently demonstrated that the catheter treatment of the pulmonic valve versus open surgery is cost saving, even with a catheter device cost five times the surgical valve (69). Due to better imaging modalities and CT reconstruction, the procedural time has currently been reduced. In general, the transfemoral procedures are done in local anesthesia and conscious sedation. 
This publication appeared at a time when it was no special DRG for TAVI, reimbursement for surgical aortic valve replacement was used, 237330 NOK (39 555 US\$). To create DRG it is necessary to have a related procedural code. The drivers and barriers of such a process are numerous and arduous both practical and political. The Edwards Sapien ${ }^{\mathrm{TM}}$ valve was the first TAVI valve receiving CE approval in 2007, and FDA approval four years later in 2011.It was only following these approvals the final DRG reimbursement rate could be established.

The first DRG designed for TAVI was in 2012: 104C=268 151 NOK, following the presentation of these data.

Health economic considerations and reimbursement decisions will play a role in TAVI expansions. We already see this effect on TAVI penetration regarding to DRG reimbursement in several European countries. Added benefits of TAVI in terms of quality of life and survival need to be documented to justify the higher costs. The large number of current and potential TAVI candidates will have a large budget impact on health care system. Therefore, both cost-effectiveness and health care budget impact studies need to be done at national levels to advice the reimbursement policy decisions. The catheter valves are not getting cheaper, the total costs go down as the patient need ICU care for a shorter time and will be discharged earlier. Due to regulations for reimbursement, Germany has the highest number of TAVI per million inhabitants of 160 , in the Nordics the number is about 40 , but increasing, and in US around 70. At present, approximately 180000 patients may be considered as TAVI candidates in EU (European Union) and Northern America annually. This number might increase up to 270000 if indications expand to intermediate and low risk patients (51).

The quality of life for this cohort was recorded, but not published in paper I, as the mortality was quite high there were many dropouts in addition to missing forms. The recording started from patient number six, that means for 45 patients. As the cohort was already small, we did not divide into a transapical and a transfemoral group. During this initial phase of inclusion, there were also a wide range of results, hence it was difficult to make any statistically significant conclusion. However, among these first included patients (6-50) there was a tendency of improvement in both physical and 
mental score for quality of life $(p<0.035$ and 0.044$)$. This was among the first patients treated for aortic stenosis with transcatheter valve at a time with limited experience in patient selection and implantation technique.

\section{Safety and efficiency of alternative access routes and devices for TAVI. Development of new} techniques

As an alternative to transfemoral access in patients with peripheral vascular disease the transapical approach was established. Moreover, for some patients neither the transfemoral nor the transapical way of acceding the aortic annulus could safely be applied, hence the transaortic approach emerges as another alternative to central approach.

Access design is an important issue in TAVI (44) and tailoring the procedure in the safest way for each individual with CT reconstruction and measurements is mandatory. More suitable for transaortic access compared to transapical are patients with no previous sternotomy, pulmonary disease, obese patients, shaggy descending aorta and aortic arch, and candidates with "soft" apex and reduced ejection fraction.

The direct aortic access is feasible and safe through right mini thoracotomy as well as mini sternotomy for selected patients. The cannulation technique of ascending aorta is well known for cardiothoracic surgeons. It seems easier to have good coaxial alignment and to position the bigger valves via this central approach than with other access routes, especially in situations with horizontal ascending aorta.

This study was conducted in the early TAVI era, within a population quite different from the TAVI population of today. Currently, there are better imaging tools for planning the procedure with more exact sizing and implantation angles, and the next generation of TAVI valves will have less paravalvular leakage and pacemaker implantation. In addition, the patients are becoming younger with less comorbidity, some of the patients treated in the early era of TAVI might today have been excluded. 
3. Relation between intra-operative improvement of the left ventricular function to long term mortality, morbidity, functional capacity and quality of life (QoL)

Our study could confirm that there was a correlation between cardiac function on echo, NYHA class and quality of life. This may be of importance for performing an optimal selection of patients for TAVI treatment and may give indication on who will benefit from this treatment.

The results of the study suggest that a preoperative test of myocardial contractile reserve might improve risk stratification and patient selection prior to procedure. The non-responders which did not improve the left ventricle longitudinal contractile function after valve implantation had a high mortality rate, showed almost no improvement in NYHA class and had only a slight improvement in SF 36 scores. They even had a deterioration of the score for Social Function and General Health, reflecting the disappointment of not getting better.

The study also addressed the importance of measuring the quality of life in addition to the physical parameters to have an impression of the total value of the treatment. The analysis showed that there was a strong correlation between all these parameters.

By classifying the patients in per operative responders and non-responders attempts were made to identify who would take advantage from the procedure.

The most economical and clinical benefit would be to identify preoperative risk factors for death and re-hospitalization.

For the responders and the non-responders, the risk factors for cardiac death and re-hospitalization were identified as EuroScore, pulmonary hypertension and the mean pressure gradient of the aortic stenosis.

Some possible differences in the patient characteristics, however, with borderline significance, were identified different in the responders vs non-responders:

A significant difference in cardiac death $(p<0.04)$ and cardiac death and re-hospitalization $(p<0.01)$ was observed for the responders compared to the non-responders. At one year $66 \%$ of the non- 
responders had either been re-hospitalized or had been dead. This was seven-fold more than among the responders.

A correlation was observed between the responder and higher quality of life score at one year follow up compared to the non-responder. There was a significant improvement in Physical function, General Health, Vitality and Social Function for the responders, but no significant difference for the non- responders which did not improve the left ventricular longitudinal contractility after the procedure.

For the total group (responders/non-responders) there was a significant improvement in the Physical Function and Emotial Role, and borderline for the Vitality, Social Function and Mental health, though, there were some missing reporting, When evaluating the data, the high mortality rate has also to be taken into consideration. These findings underline the need for a better selection of patients for the procedure, as all survivors had a significant improvement of quality of life.

The cost of the device was the same in both groups, consequently the value of healthcare of treating the responders compared to the non-responders was higher. The difference in RE (role-emotional) which was quite high, was almost the same for both groups. This could reflect to some extent a "sham effect" of the procedure, the patient felt taken care of and well treated. The responders had all over improvement in all the subgroups of quality of life, whereas the non- responders had only a slight increase in the quality of life factors, and even a decrease in social function and general health. This way to evaluate the value of health care of a new treatment option may have a major implication for health care resource planning in the future.

4. Radiation burden on the patient and the staff involved in the procedure. New software and equipment for echo and CT reconstruction are evolving. CT allows for an exact sizing and calculation of implantation angle and is today mandatory in the planning of treatment with transcatheter procedures. Echo and CT planning reduce the need of contrast volume to be used and the radiation time during the procedure both for implanters and patients. 
In fluoroscopic procedures, the variation in occupational radiation doses usually relates to the variation in patient doses. In our study, a greater variation in operator doses was observed, depending on the position at the procedural table and access. In addition, fluoroscopy time, fluoroscopy pulse rate, tight collimation and shielding, the distance between operator and entry point of the $x$-ray beam on the patient were all of vital importance when estimation the occupational doses. The inverse square law explains how the intensity of radiation varies as the inverse square of the distance from an isotopic source.

During transaortic access, the cardiothoracic surgeons were positioned close to the patient's heart, and near the edge of the $\mathrm{x}$-ray beam. They need free access to the ascending aorta.

The eye lens dose was the limiting dose. It has been suggested that surgeons should make use of protective eye wear and to perform only a restricted annual number of procedures. Following this investigation several improvements have been established to limit the radiation dose. Pre-calculation of implant angle, new and better delivery systems for the transcatheter heart valves and experience have also reduced the radiation time. This study concludes the same as studies for $\mathrm{PCl}$ after introducing radial artery access for angiography where the operator is close to the radiation source and patient. Special equipment is designed for protection, but unfortunately not very suitable. There are some covering sheaths protecting the operator, but "enclose" the radiation to the patient. For surgeons it is also an issue with the radiation of fingers and hands. Nevertheless, in this study no measurements of the radiation dose for the exposure of hands were performed as such equipment was not available.

This is important since the numbers of TAVI procedures are increasing. Currently, a smaller number of procedures are done with central access which cause the highest exposition of radiation doses and may especially harm the eye lens. Still the occupational dose has to be addressed and implanters should particularly protect the eyes.

The numbers of TAVI procedures are still increasing and indications expanding. Even though most of the procedures are performed transfemorally and the occupational radiation doses are decreasing 
for each procedure, one should keep this in mind and look for alternative imaging modalities for the periprocedural guidance. New echo software may be a solution, but for the transesophageal echocardiography the patients may need to be in general anesthesia. Specific software for eyetracking has been developed. This may increase the awareness of the operators of the fluoroscopic time and prevent unnecessary use of fluoroscopy when not looking to the screen. There must be a balance between the imaging modality and "least invasiveness".

\section{TAVI MANAGEMENT FOLLOWING THIS STUDY}

Since the release to marked, the prizing of the transcatheter valves has been of an important issue, as has been the question of who should perform the procedure. The different specialties seem to merge. The interventional cardiologists and radiologists were traditionally most familiar with fluoroscopic-guided procedures and the surgeons with valve replacement. Surgical experience is necessary for femoral cut down and central access such as transapical and transaortic implantation. Some surgeons have learned the catheter techniques for percutaneous transfemoral access. In a severely calcified aortic annulus rupture may occur as the valve is deployed and pericardial tamponade may occur, requiring urgent opening of the chest. Thus, guidelines and insurance requirements advocate cross-disciplinary approach where the cardiac surgeons and cardiologists work together during the procedure in what is called "Heart team". The concept of hybrid room provided with surgical as well as interventional instruments has been lounged, creating safe environment where both advanced interventions and open surgery may be performed. The upcoming discussion is the same as when percutaneous coronary intervention $(\mathrm{PCl})$ was introduced in the 1970s very well discussed by Michael Mack already in 2008 in the paper "Fool me once, shame on you; fool me twice, shame on me! A perspective on the emerging world of percutaneous valve therapy"(65). Recently his group published a concern regarding surgeons not being involved in 
transcatheter valve therapy, where the solution is to formalize and structurize the education in catheter skills for surgeons. This is to keep the Heart Team concept valid (66).

Machine learning is a new discipline in computer sciences aiming to predict outcomes of complex dataset using algorithms that interactively learn from data (61-63). Machine learning methods can generate robust models to predict in-hospital mortality for transcatheter aortic valve implantations (70). Rather than considering fixed assumptions on data behavior and variable preselection, machine learning algorithms allow the data to create the model by detecting the learning underlying patterns. To predict adverse cardiovascular events, this concept has already been introduced in cardiovascular studies (61-63).

Moreover, the new interventional approach may induce a radiation hazard to the staff, particularly when surgeons work almost directly under the image enhancer when the central approach is used. Currently a paradigm shift in medical reimbursement from quantity-based fee-for-service payment models to quality-based bundled-payment models. This may have the consequence that some highrisk patients are denied care in secondary institutions in fear of postoperative complications and referred to academic tertiary centers. This may potentially impose financial deficits in the latter institutions. Surgeons remain under pressure to decrease costs, but they may not be able to comply with these requirements. To keep the value surgeons must perform excellent standard and service, advantageous to the patients, but may limit innovation and medical research. Transforming the health care from volume- to value based medicine will require major changes in quantification of costs and quality measures to reshape research and education.

\section{Expanding the indications}

Transcatheter valve treatment is now offered to younger patients with less comorbidity, though still the durability of these valves has to be been proven. Bioprostheses are prone to structural valve degeneration resulting in limited long-term durability. For biological surgical valves the degeneration may appear abruptly at a few years after implantation, especially in small dimensions and younger 
patients. While in other cases the bioprostheses may last for $15-20$ years (71). The empirical fact that catheter valves are as durable as surgical aortic valve replacements is still debatable.

Technology is emerging and only the fantasy may limit the use of the catheter valve techniques. The original aortic catheter valves ("TAVI valves") are also used for valve-in-valve aortic and for the valvein-valve/ring mitral and tricuspid as well as for implantation in situations such as mitral annular calcification

By expanding the indications to younger patients (72) other unresolved problems may occur:

1) Bicuspid aortic valve was until recently a contraindication for TAVI because of increased risk of paravalvular leakage and need of permanent pacemaker. The problem is the shape and uneven distribution of and only two cusps cause difficulties in sizing, and strong radial force must be applied to make the valve circular. There are promising results with special designed valves for this purpose $(15,73)$.

2) Aortic regurgitation has so far been a contraindication for TAVI, but growing experience and/ or special designed valve make this possible (74-76).

3) Valve-in-valve has become "the default treatment" for redo procedures in degenerated biological valves $(77,78)$. There are however some limitations to use this technique in the smaller surgical valves and in the bioprostheses with cusps mounted exteriorly on the valve stent in concern of coronary occlusion. A suggested solution may be the valve cracking technique (79-82) and the BASILICA (Bioprosthetic or native Aortic Scallop Intentional Laceration to prevent latrogenic Coronary Artery obstruction during TAVI) (83-85) procedure. The question of patient prosthesis mismatch also may arise, about $20 \%$ of TAVI procedures have too small valve implanted and patient prosthesis mismatch may occur (86).

4) New pacemaker rate post TAVI is around $12 \%$ according to the latest report from the TCT registry, some variations depending on device. A State of the art report of Auffret et al from 2017 report 12$22 \%$ for Edwards SAPIEN S3 valve and $18-65 \%$ for the CoreValve ${ }^{\mathrm{TM}}$ system (87). It seems like the rate of pacemaker need is dependent on the degree of paravalvular leak. The more severe paravalvular 
leak, the more pacemakers need to be implanted $(88,89)$. Longstanding ventricular pacing may after some time reduce the left ventricular ejection fraction accordingly increases the risk of heart failure, not of big concern in the elderly patient population, but may be of greater importance in younger patients with a much longer life expectancy (90).

5) An important publication from Sinning et al (91) proved the importance of paravalvular leak regarding long time survival and defined the aortic regurgitant index, AR index $=($ DBP-LVEDP $) / S B P$, DBP: diastolic blood pressure, LVEDP: left ventricular end diastolic pressure, SBP: systolic blood pressure. Aortic regurgitation -index above 25 indicates small paravalvular leak and aortic regurgitation index below 25 indicates severe paravalvular leak. This again will have great influence on survival. With aortic regurgitation index above 25 one year survival is $83.5 \%$ vs $54.0 \%$ with index below 25 (91).

6) Transcatheter heart valves have been shown to be more strongly associated with leaflet thickening than surgical valves. This phenomenon may possibly accelerate the risk of structural valve deterioration (SVD) $(92,93)$. Moreover, one has to make a universal agreement on the definition of structural valve degeneration. The surgical valves have been followed up with the end point "need of reoperation". In the era of TAVI it is different. "Standardized definition of structural valve degeneration for surgical and transcatheter bioprosthetic heart valves" published by Dvir et al (93) is emphasizing the definition of structural valve deterioration on echo criteria and makes suggestions to clinical approach. Studies on transcatheter valve durability are warranted. 


\section{ORGANIZATION AND TECHNOLOGY FOR MINIMAL INVASIVE}

\section{TREATMENT OF STRUCTURAL HEART DISEASE}

Several challenges to emphasize when implementing a new program into clinical practice have been outlined in this Thesis, some of them are:

- Building new teams, relocating roles and redefine specialties

- Economical and political issues within the hospital and in the society in general

- In-hospital logistics

- Patient selection

- Procedural planning, new imaging modalities

- Feasibility and safety

- Ethics

The most essential when starting a cross-disciplinary program is the ability of collaboration between specialties (cardiology/radiology/surgery) such as in the "Heart Team" of TAVI, and to define "the ingroup structure" in order to provide good decision making for the patient and not promoting the "brilliance of the operators". A strong leadership is essential. A reasonable way of starting is to have a "neutral playground" which is not involved in the daily routine work. The doctors involved will not be on their "home ground" and procedural time will not be any issue. In our centre there have been conducted several psychosocial studies on the process of building up an innovative medical procedure as we recognize in TAVI with many cross disciplinary specialties in a common arena such as the hybrid operation room (94). Davide Nicolini et al found that a "phenomenon" like TAVI requires that there is a modification of the traditional views of expertise to acknowledge its social, material and distributed nature. Furthermore, in the case of TAVI, expertise feeds upon the broad circuits of knowledge created by the combination of professional relationships, social ties and economic interests. 
To organize the TAVI treatment in a hybrid operation room seems to be the safest and most convenient way both for the patient and the personnel. A hybrid room has the equipment to save the patient if complications occur, i.e. conversion to surgery from catheter treatment is possible. Recent FDA approval will make this TAVI treatment available also in younger and low risk patients. However, the tolerance for failure must be very restricted, and no serious adverse events have to be accepted as long as the "golden standard" treatment of surgical aortic valve replacement can be performed with a minimum of risk and complications.

\section{LIMITATIONS}

This clinical trial is based on a prospective consecutive inclusion of patients and is not a randomized study. The implementation of this new method of TAVI had first to be proven feasible and safe. When introducing this new technology of TAVI, the indications had to be set, hence during the first years the number of patients enrolled in the TAVI clinical trial at OUS was very limited, Figure7.

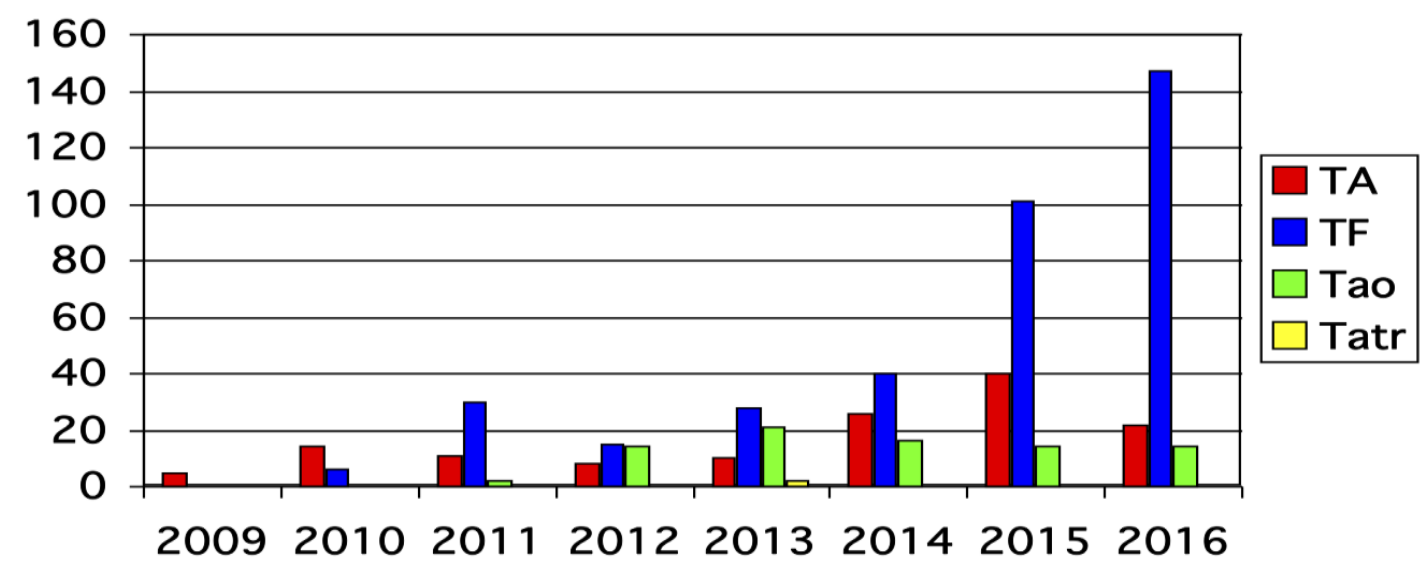

Figure 7. The number of procedures and access routes used for TAVI at Rikshospitalet from 2009 to 2016.TA: transapical, TF: transfemoral, Tao: transaortic, Tat: transatrial. 
For the recording of quality of life, there were many drop outs due to incomplete patient questionnaires also due to high one year mortality. For the sub groups Period 1 and Period 2 there was a wide range of the scoring values which might have an influence on the statistical analysis.

\section{CONCLUSIONS}

This study has concentrated on several aspects of safety and value based medical health care when introducing this high technology such as TAVI into clinical practice. There are several implications affecting the individual patient, the hospital, the society and the financial burden this may cause, as well as having an impact on the heart team members.

1. In 2011-2012 there were no special TAVI reimbursement and therefore a significant discrepancy between reimbursement and actual costs. This was also during the implementation period of new technology and precautions to ensure safety had to be taken, extending the procedural time and the length of stay. If the study had been performed today, the costs had most probably been covered by shorter stay and sufficient DRG reimbursement despite that the costs of devices have remained almost unchanged.

2. The direct aorta technique for TAVI is safe and feasible for both the Edwards Sapien ${ }^{\text {TM }}$ system and the CoreValve ${ }^{\mathrm{TM}}$ system and is an alternative to transapical access for selected patients.

3. The immediate response to TAVI with better contractility of the myocardium predicts the clinical effect of the treatment. The responders had less adverse events and longer survival than the non-responders. The responders also had better improvement in quality of life. The use of tissue Doppler imaging to evaluate myocardial response may improve risk stratification and patient selection for TAVI and identify which patients should not be treated. 
4. Radiation burden, especially to the eye lens, may be the limiting factor for TAVI implanters, especially for central TAVI. Reduction in procedural time, led protecting glasses and supplementary imaging modalities will reduce the radiation exposure.

5. The quality of life assessment is important to evaluate the value of a new treatment together with the morbidity and mortality records. Only these factors can estimate the value of the interventional treatment for the patient and also justify cost and mortality to the health authorities.

Our study shows the importance of recording the results and hazards from day one when implementing a new method into clinical practice, otherwise it is very difficult to justify a treatment method with $20 \%$ one year mortality. New devices are coming to the marked, at present most attention has been paid to the transcatheter treatment of tricuspid- and mitral valve disease. For these valves the imaging for procedural planning is even more important and sophisticated. The safety for the patient must always come first in addition to avoiding hazards for the therapists. The logistics, procedural planning, procedural performance in safe surroundings by a well-functioning heart team and a database for the documentation of the benefit for the patient, have to be in place from the onset of the TAVI program. 


\section{REFERENCES}

1. Holmes DR, Jr., Califf R, Farb A, Abel D, Mack M, Syrek Jensen T, et al. Overcoming the Challenges of Conducting Early Feasibility Studies of Medical Devices in the United States. J Am Coll Cardiol. 2016;68(17):1908-15.

2. Dearani JA, Rosengart TK, Marshall MB, Mack MJ, Jones DR, Prager RL, et al. Incorporating Innovation and New Technology Into Cardiothoracic Surgery. Ann Thorac Surg. 2019;107(4):1267-74. 3. Vaslef SN, Roberts WC. Early descriptions of aortic valve stenosis. Am Heart J. 1993;125(5 Pt 1):1465-74.

4. Sgantzos M, Tsoucalas G, Markatos K, Giatsiou S, Androutsos G. Lazare Riviere (1589-1655 AD), the Pioneer Pharmacologist, Anatomist, and Surgeon, Who Gave the First Modern Description of an Aortic Valve Failure. Surg Innov. 2015;22(5):546-7.

5. Smith DE, Matthews MB. Aortic valvular stenosis with coarctation of the aorta, with special reference to the development of aortic stenosis upon congenital bicuspid valves. Br Heart J. 1955;17(2):198-206.

6. McGinn S, White PD. Clinical Observations on Aortic Stenosis. Trans Am Clin Climatol Assoc. 1933;49:132-44.

7. lung B, Baron G, Butchart EG, Delahaye F, Gohlke-Barwolf C, Levang OW, et al. A prospective survey of patients with valvular heart disease in Europe: The Euro Heart Survey on Valvular Heart Disease. Eur Heart J. 2003;24(13):1231-43.

8. Bloomstein LZ, Gielchinsky I, Bernstein AD, Parsonnet V, Saunders C, Karanam R, et al. Aortic valve replacement in geriatric patients: determinants of in-hospital mortality. Ann Thorac Surg. 2001;71(2):597-600.

9. Probst V, Le Scouarnec S, Legendre A, Jousseaume V, Jaafar P, Nguyen JM, et al. Familial aggregation of calcific aortic valve stenosis in the western part of France. Circulation. 2006;113(6):856-60.

10. Abdulkareem N, Smelt J, Jahangiri M. Bicuspid aortic valve aortopathy: genetics, pathophysiology and medical therapy. Interact Cardiovasc Thorac Surg. 2013;17(3):554-9.

11. Lindman BR, Clavel MA, Mathieu P, lung B, Lancellotti P, Otto CM, et al. Calcific aortic stenosis. Nature reviews Disease primers. 2016;2:16006.

12. Tung $M$, Wang $X$, Li F, Wang H, Guo Y, Wang C, et al. A versatile transapical device for aortic valvular disease: One-year outcomes of a multicenter study on the J-Valve system. J Cardiol. 2018.

13. Liu XB, Jiang JB, Zhou QJ, Pu ZX, He W, Dong AQ, et al. Evaluation of the safety and efficacy of transcatheter aortic valve implantation in patients with a severe stenotic bicuspid aortic valve in a Chinese population. Journal of Zhejiang University Science B. 2015;16(3):208-14.

14. lung B, Vahanian A. Epidemiology of valvular heart disease in the adult. Nat Rev Cardiol. 2011;8(3):162-72.

15. Yoon SH, Maeno Y, Kawamori H, Miyasaka M, Nomura T, Ochiai T, et al. Diagnosis and Outcomes of Transcatheter Aortic Valve Implantation in Bicuspid Aortic Valve Stenosis.

Interventional cardiology (London, England). 2018;13(2):62-5.

16. Ridley $\mathrm{CH}$, Vallabhajosyula P, Bavaria JE, Patel PA, Gutsche JT, Shah R, et al. The Sievers Classification of the Bicuspid Aortic Valve for the Perioperative Echocardiographer: The Importance of Valve Phenotype for Aortic Valve Repair in the Era of the Functional Aortic Annulus. J Cardiothorac Vasc Anesth. 2016;30(4):1142-51.

17. Rosenhek R, Zilberszac R, Schemper M, Czerny M, Mundigler G, Graf S, et al. Natural history of very severe aortic stenosis. Circulation. 2010;121(1):151-6.

18. Carabello BA, Paulus WJ. Aortic stenosis. Lancet. 2009;373(9667):956-66.

19. Baumgartner H, Falk V, Bax JJ, De Bonis M, Hamm C, Holm PJ, et al. 2017 ESC/EACTS

Guidelines for the management of valvular heart disease. Eur Heart J. 2017;38(36):2739-91. 
20. Cohn LH. The first successful surgical treatment of mitral stenosis: the 70th anniversary of Elliot Cutler's mitral commissurotomy. Ann Thorac Surg. 1993;56(5):1187-90.

21. Glover RP, Gadboys HL. Seven years' experience with transventricular aortic commissurotomy. J Thorac Surg. 1958;36(6):839-56.

22. Hufnagel CA, Harvey WP. The surgical correction of aortic regurgitation preliminary report. Bull Georgetown Univ Med Cent. 1953;6(3):60-1.

23. Kortz $A B$, Swan H. Direct vision trans-aortic approach to the aortic valve during hypothermia; experimental observations and report of successful clinical case. Ann Surg. 1956;144(2):205-14.

24. Gibbon JH, Jr. Extracorporeal maintenance of cardiorespiratory functions. Harvey Lect. 1957;53:186-224.

25. Gott VL, Alejo DE, Cameron DE. Mechanical heart valves: 50 years of evolution. Ann Thorac Surg. 2003;76(6):S2230-9.

26. Grunkemeier GL, Starr A. Twenty-five year experience with Starr-Edwards heart valves: follow-up methods and results. Can J Cardiol. 1988;4(7):381-5.

27. Storstein O, Efskind L. Aortic stenosis. Diagnosis and surgical treatment. Cardiologia. 1966;49(5):259-69.

28. Pollock SG, Dent JM, Simek CL, Gimple LW, Johns RA, Kaul S, et al. Starr-Edwards valve thrombosis detected preoperatively by transesophageal echocardiography. Cathet Cardiovasc Diagn. 1994;31(2):156-7.

29. Shaikh A, Jamil Tajik A. Excellence and Durability: A Normally Functioning Bjork-Shiley FlatDisc Prosthesis 42 Years Post Implantation. J Heart Valve Dis. 2016;25(2):237-9.

30. Kvitting JP, Geiran OR. Karl Viktor Hall: From In Situ Vein Bypass to the Tilting Disc Heart Valve Prosthesis. Ann Thorac Surg. 2016;102(5):1756-61.

31. Carpentier A, Deloche A, Relland J, Fabiani JN, Forman J, Camilleri JP, et al. Six-year follow-up of glutaraldehyde-preserved heterografts. With particular reference to the treatment of congenital valve malformations. J Thorac Cardiovasc Surg. 1974;68(5):771-82.

32. Chikwe J, Filsoufi F. Durability of tissue valves. Semin Thorac Cardiovasc Surg. 2011;23(1):18-

23.

33. Brown JM, O'Brien SM, Wu C, Sikora JA, Griffith BP, Gammie JS. Isolated aortic valve replacement in North America comprising 108,687 patients in 10 years: changes in risks, valve types, and outcomes in the Society of Thoracic Surgeons National Database. J Thorac Cardiovasc Surg. 2009;137(1):82-90.

34. Culler SD, Cohen DJ, Brown PP, Kugelmass AD, Reynolds MR, Ambrose K, et al. Trends in Aortic Valve Replacement Procedures Between 2009 and 2015: Has Transcatheter Aortic Valve Replacement Made a Difference? Ann Thorac Surg. 2018;105(4):1137-43.

35. Martens S, Ploss A, Sirat S, Miskovic A, Moritz A, Doss M. Sutureless aortic valve replacement with the $3 f$ Enable aortic bioprosthesis. Ann Thorac Surg. 2009;87(6):1914-7.

36. Meuris B, Flameng WJ, Laborde F, Folliguet TA, Haverich A, Shrestha M. Five-year results of the pilot trial of a sutureless valve. J Thorac Cardiovasc Surg. 2015;150(1):84-8.

37. Pfeiffer S, Fischlein T, Santarpino G. Sutureless Sorin Perceval Aortic Valve Implantation. Semin Thorac Cardiovasc Surg. 2017;29(1):1-7.

38. Borger MA, Dohmen P, Misfeld M, Mohr FW. Current trends in aortic valve replacement: development of the rapid deployment EDWARDS INTUITY valve system. Expert Rev Med Devices. 2013;10(4):461-70.

39. Concistre G, Chiaramonti F, Santarpino G, Pfeiffer S, Marchi F, Vogt F, et al. Left ventricular mass regression after two alternative sutureless aortic bioprostheses. Innovations (Philadelphia, $\mathrm{Pa}$ ). 2015;10(2):114-9.

40. Barnhart GR, Shrestha ML. Current Clinical Evidence on Rapid Deployment Aortic Valve Replacement: Sutureless Aortic Bioprostheses. Innovations (Philadelphia, Pa). 2016;11(1):7-14.

41. Doenst T, Amorim PA, Al-Alam N, Lehmann S, Mukherjee C, Faerber G. Where is the common sense in aortic valve replacement? A review of hemodynamics and sizing of stented tissue valves. J Thorac Cardiovasc Surg. 2011;142(5):1180-7. 
42. Durko AP, Head SJ, Pibarot P, Atluri P, Bapat V, Cameron DE, et al. Characteristics of surgical prosthetic heart valves and problems around labelling: a document from the European Association for Cardio-Thoracic Surgery (EACTS)-The Society of Thoracic Surgeons (STS)-American Association for Thoracic Surgery (AATS) Valve Labelling Task Force. Eur J Cardiothorac Surg. 2019;55(6):1025-36.

43. Cribier A, Eltchaninoff H, Bash A, Borenstein N, Tron C, Bauer F, et al. Percutaneous transcatheter implantation of an aortic valve prosthesis for calcific aortic stenosis: first human case description. Circulation. 2002;106(24):3006-8.

44. Biasco L, Ferrari E, Pedrazzini G, Faletra F, Moccetti T, Petracca F, et al. Access Sites for TAVI: Patient Selection Criteria, Technical Aspects, and Outcomes. Frontiers in cardiovascular medicine. 2018;5:88.

45. Duran A, Hian SK, Miller DL, Le Heron J, Padovani R, Vano E. Recommendations for occupational radiation protection in interventional cardiology. Catheter Cardiovasc Interv. 2013;82(1):29-42.

46. The 2007 Recommendations of the International Commission on Radiological Protection. ICRP publication 103. Ann ICRP. 2007;37(2-4):1-332.

47. Werner GS. Reducing radiation exposure during $\mathrm{PCl}$ of chronic total occlusions - better is not good enough. Eurolntervention. 2018;14(5):e496-e8.

48. Colleran R, Douglas PS, Hadamitzky M, Gutberlet M, Lehmkuhl L, Foldyna B, et al. An FFRCT diagnostic strategy versus usual care in patients with suspected coronary artery disease planned for invasive coronary angiography at German sites: one-year results of a subgroup analysis of the PLATFORM (Prospective Longitudinal Trial of FFRCT: Outcome and Resource Impacts) study. Open heart. 2017;4(1):e000526.

49. Douglas PS, Pontone G, Hlatky MA, Patel MR, Norgaard BL, Byrne RA, et al. Clinical outcomes of fractional flow reserve by computed tomographic angiography-guided diagnostic strategies vs. usual care in patients with suspected coronary artery disease: the prospective longitudinal trial of FFR(CT): outcome and resource impacts study. Eur Heart J. 2015;36(47):3359-67.

50. Fernandes P, Cleland A, Bainbridge D, Jones PM, Chu MW, Kiaii B. Development of our TAVI protocol for emergency initiation of cardiopulmonary bypass. Perfusion. 2015;30(1):34-9.

51. Durko AP, Osnabrugge RL, Van Mieghem NM, Milojevic M, Mylotte D, Nkomo VT, et al. Annual number of candidates for transcatheter aortic valve implantation per country: current estimates and future projections. Eur Heart J. 2018;39(28):2635-42.

52. Leon MB, Nikolsky E. The next revolution: percutaneous aortic valve replacement. Rambam Maimonides medical journal. 2010;1(2):e0016.

53. Patel AA, Donegan D, Albert T. The 36-item short form. J Am Acad Orthop Surg. 2007;15(2):126-34.

54. Drudi LM, Ades M, Turkdogan S, Huynh C, Lauck S, Webb JG, et al. Association of Depression With Mortality in Older Adults Undergoing Transcatheter or Surgical Aortic Valve Replacement. JAMA cardiology. 2018;3(3):191-7.

55. Porter ME. What is value in health care? N Engl J Med. 2010;363(26):2477-81.

56. Glotzbach JP, Sharma V, Tonna JE, Pettit JC, McKellar SH, Eckhauser AW, et al. Value-driven cardiac surgery: Achieving "perfect care" after coronary artery bypass grafting. J Thorac Cardiovasc Surg. 2018.

57. Mishra V, Skeie B, Vaaler S, Amlie E. A comparison of actual registered costs and costs derived from diagnosis-related groups (DRGs) for patients undergoing heart transplantation, lung transplantation, and thoracotomy for other lung diseases. Transpl Int. 2001;14(6):361-9.

58. Skeie B, Mishra V, Vaaler S, Amlie E. A comparison of actual cost, DRG-based cost, and hospital reimbursement for liver transplant patients. Transpl Int. 2002;15(9-10):439-45.

59. Mack MJ, Leon MB, Thourani VH, Makkar R, Kodali SK, Russo M, et al. Transcatheter AorticValve Replacement with a Balloon-Expandable Valve in Low-Risk Patients. N Engl J Med. 2019;380(18):1695-705. 
60. Popma JJ, Deeb GM, Yakubov SJ, Mumtaz M, Gada H, O'Hair D, et al. Transcatheter AorticValve Replacement with a Self-Expanding Valve in Low-Risk Patients. N Engl J Med.

2019;380(18):1706-15.

61. Deo RC. Machine Learning in Medicine. Circulation. 2015;132(20):1920-30.

62. Shouval R, Hadanny A, Shlomo N, lakobishvili Z, Unger R, Zahger D, et al. Machine learning for prediction of 30-day mortality after ST elevation myocardial infraction: An Acute Coronary Syndrome Israeli Survey data mining study. Int J Cardiol. 2017;246:7-13.

63. Shouval R, Bondi O, Mishan H, Shimoni A, Unger R, Nagler A. Application of machine learning algorithms for clinical predictive modeling: a data-mining approach in SCT. Bone Marrow Transplant. 2014;49(3):332-7.

64. Sardar P, Abbott JD, Kundu A, Aronow HD, Granada JF, Giri J. Impact of Artificial Intelligence on Interventional Cardiology: From Decision-Making Aid to Advanced Interventional Procedure Assistance. JACC Cardiovasc Interv. 2019;12(14):1293-303.

65. Mack M. Fool me once, shame on you; fool me twice, shame on me! A perspective on the emerging world of percutaneous heart valve therapy. J Thorac Cardiovasc Surg. 2008;136(4):816-9. 66. Nguyen TC, Tang GHL, Nguyen S, Forcillo J, George I, Kaneko T, et al. The train has left: Can surgeons still get a ticket to treat structural heart disease? J Thorac Cardiovasc Surg. 2019;157(6):2369-76.e2.

67. Biancari F, Rosato S, D'Errigo P, Ranucci M, Onorati F, Barbanti M, et al. Immediate and Intermediate Outcome After Transapical Versus Transfemoral Transcatheter Aortic Valve Replacement. Am J Cardiol. 2016;117(2):245-51.

68. Hernandez J, Machacz SF, Robinson JC. US hospital payment adjustments for innovative technology lag behind those in Germany, France, and Japan. Health Aff (Millwood). 2015;34(2):26170 .

69. Andresen B, Mishra V, Lewandowska M, Andersen JG, Andersen MH, Lindberg H, et al. Inhospital cost comparison between percutaneous pulmonary valve implantation and surgery. Eur $\mathrm{J}$ Cardiothorac Surg. 2017;51(4):747-53.

70. Hernandez-Suarez DF, Kim Y, Villablanca P, Gupta T, Wiley J, Nieves-Rodriguez BG, et al. Machine Learning Prediction Models for In-Hospital Mortality After Transcatheter Aortic Valve Replacement. JACC Cardiovasc Interv. 2019;12(14):1328-38.

71. Capodanno D, Petronio AS, Prendergast B, Eltchaninoff $H$, Vahanian A, Modine $T$, et al. Standardized definitions of structural deterioration and valve failure in assessing long-term durability of transcatheter and surgical aortic bioprosthetic valves: a consensus statement from the European Association of Percutaneous Cardiovascular Interventions (EAPCI) endorsed by the European Society of Cardiology (ESC) and the European Association for Cardio-Thoracic Surgery (EACTS). Eur Heart J. 2017;38(45):3382-90.

72. De Backer O, Sondergaard L. Challenges When Expanding Transcatheter Aortic Valve Implantation to Younger Patients. Frontiers in cardiovascular medicine. 2018;5:45.

73. Frangieh AH, Michel J, Deutsch $\mathrm{O}$, Joner $\mathrm{M}$, Pellegrini $\mathrm{C}$, Rheude $\mathrm{T}$, et al. Aortic annulus sizing in stenotic bicommissural non-raphe-type bicuspid aortic valves: reconstructing a three-dimensional structure using only two hinge points. Clin Res Cardiol. 2018.

74. Spina R, Anthony C, Muller DW, Roy D. Transcatheter Aortic Valve Replacement for Native Aortic Valve Regurgitation. Interventional cardiology (London, England). 2015;10(1):49-54.

75. Bob-Manuel T, Kadire S, Heckle MR, Wang J, Ibebuogu UN. Outcomes following transcatheter aortic valve replacement in patients with native aortic valve regurgitation. Annals of translational medicine. 2018;6(1):8.

76. Hanson ID, Dalal PK, Renard BM, Hanzel GS, Vivacqua A. Emergency Valve-in-Valve Transcatheter Aortic Valve Implantation for the Treatment of Acute Stentless Bioprosthetic Aortic Insufficiency and Cardiogenic Shock. Case reports in cardiology. 2018;2018:6872748.

77. Dvir D, Webb JG. Transcatheter aortic valve-in-valve implantation for patients with degenerative surgical bioprosthetic valves. Circ J. 2015;79(4):695-703. 
78. Webb JG, Murdoch D, Dvir D. Will Transcatheter Replacement Become the New Default Therapy When Bioprosthetic Valves Fail? J Am Coll Cardiol. 2018;72(4):383-5.

79. Allen KB, Chhatriwalla AK, Cohen DJ, Saxon JT, Aggarwal S, Hart A, et al. Bioprosthetic Valve Fracture to Facilitate Transcatheter Valve-in-Valve Implantation. Ann Thorac Surg. 2017;104(5):15018.

80. Chhatriwalla AK, Allen KB, Saxon JT, Cohen DJ, Aggarwal S, Hart AJ, et al. Bioprosthetic Valve Fracture Improves the Hemodynamic Results of Valve-in-Valve Transcatheter Aortic Valve Replacement. Circ Cardiovasc Interv. 2017;10(7).

81. Allen KB, Chhatriwalla AK, Saxon JT, Cohen DJ, Nguyen TC, Webb J, et al. Bioprosthetic valve fracture: Technical insights from a multicenter study. J Thorac Cardiovasc Surg. 2019.

82. Sathananthan J, Sellers S, Barlow AM, Stanova V, Fraser R, Toggweiler S, et al. Valve-in-Valve Transcatheter Aortic Valve Replacement and Bioprosthetic Valve Fracture Comparing Different Transcatheter Heart Valve Designs: An Ex Vivo Bench Study. JACC Cardiovasc Interv. 2019;12(1):6575.

83. Lederman RJ, Babaliaros VC, Rogers T, Khan JM, Kamioka N, Dvir D, et al. Preventing Coronary Obstruction During Transcatheter Aortic Valve Replacement: From Computed Tomography to BASILICA. JACC Cardiovasc Interv. 2019;12(13):1197-216.

84. Khan JM, Greenbaum AB, Babaliaros VC, Rogers T, Eng MH, Paone G, et al. The BASILICA Trial: Prospective Multicenter Investigation of Intentional Leaflet Laceration to Prevent TAVR Coronary Obstruction. JACC Cardiovasc Interv. 2019;12(13):1240-52.

85. Dvir D, Khan J, Kornowski R, Komatsu I, Chatriwalla A, Mackenson GB, et al. Novel strategies in aortic valve-in-valve therapy including bioprosthetic valve fracture and BASILICA. Eurolntervention. 2018;14(Ab):Ab74-ab82.

86. Schofer N, Deuschl F, Rubsamen N, Skibowski J, Seiffert M, Voigtlander L, et al. Prosthesispatient mismatch after transcatheter aortic valve implantation: prevalence and prognostic impact with respect to baseline left ventricular function. Eurolntervention. 2019;14(16):1648-55.

87. Auffret V, Puri R, Urena M, Chamandi C, Rodriguez-Gabella T, Philippon F, et al. Conduction Disturbances After Transcatheter Aortic Valve Replacement: Current Status and Future Perspectives. Circulation. 2017;136(11):1049-69.

88. Maeno $\mathrm{Y}$, Abramowitz $\mathrm{Y}$, Kawamori H, Kazuno $\mathrm{Y}$, Kubo S, Takahashi N, et al. A Highly Predictive Risk Model for Pacemaker Implantation After TAVR. JACC Cardiovasc Imaging. 2017;10(10 Pt A):1139-47.

89. Al-Azzam F, Greason KL, Krittanawong C, Williamson EE, McLeod CJ, King KS, et al. The influence of native aortic valve calcium and transcatheter valve oversize on the need for pacemaker implantation after transcatheter aortic valve insertion. J Thorac Cardiovasc Surg. 2017;153(5):105662.e1.

90. Campelo-Parada F, Nombela-Franco L, Urena M, Regueiro A, Jimenez-Quevedo P, Del Trigo $M$, et al. Timing of Onset and Outcome of New Conduction Abnormalities Following Transcatheter Aortic Valve Implantation: Role of Balloon Aortic Valvuloplasty. Revista espanola de cardiologia (English ed). 2018;71(3):162-9.

91. Sinning JM, HammerstingI C, Vasa-Nicotera M, Adenauer V, Lema Cachiguango SJ, Scheer AC, et al. Aortic regurgitation index defines severity of peri-prosthetic regurgitation and predicts outcome in patients after transcatheter aortic valve implantation. J Am Coll Cardiol. 2012;59(13):1134-41.

92. Makkar RR, Fontana G, Jilaihawi H, Chakravarty T, Kofoed KF, De Backer O, et al. Possible Subclinical Leaflet Thrombosis in Bioprosthetic Aortic Valves. N Engl J Med. 2015;373(21):2015-24.

93. Dvir D, Bourguignon T, Otto CM, Hahn RT, Rosenhek R, Webb JG, et al. Standardized Definition of Structural Valve Degeneration for Surgical and Transcatheter Bioprosthetic Aortic Valves. Circulation. 2018;137(4):388-99.

94. Olsen PI, Mork BE, Hoholm T, Nicolini D. How global medical innovation processes evolve: The case of Transcatheter Aortic Valve Implantation. Academy of Management Proceedings. 2018;2018(1):16917. 
PAPERS 



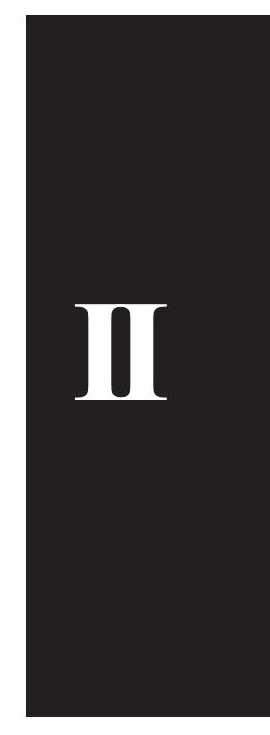





\title{
Direct Aorta Ascending Approach in Transcatheter Aortic Valve Implantation
}

\author{
Gry Dahle, MD, and Kjell-Arne Rein, MD, PhD
}

\begin{abstract}
Objective: Direct aorta ascending approach [transaortic approach (TAo)] is a new access way for transcatheter aortic valve implantation (TAVI) to be used in patients with peripheral vascular disease and as an alternative to transapical approach.

Methods: Both the Edwards SAPIEN and CoreValve systems were used. Depending on the position of the ascending aorta, the relation to the sternum, the brachiocephalic vein, vein grafts, and left and right internal mammary artery grafts from previous heart surgery, either right minithoracotomy or left ministernotomy was selected. Computed tomographic scan with reconstruction was applied for this decision making. A hybrid operating room was used with echocardiographic and fluoroscopy guidance.
\end{abstract}

Results: We have done 30 procedures via the aorta ascendens. The mean age of the patients was 80 years, and 18 were men. The mean Logistic EuroSCORE of 33 reflects the comorbidities. More than half of the patients had coronary vessel disease and had undergone coronary artery bypass graft; $20 \%$ had abdominal aortic aneurysm. The mean ejection fraction was $41 \%$, and the patients were in New York Heart Association class III to IV. The mean gradient was $50 \mathrm{~mm} \mathrm{Hg}$, and the mean valve area was $0.7 \mathrm{~cm}^{2}$. The mean valve size was $28 \mathrm{~mm}$. The use of Edwards SAPIEN versus CoreValve was $50 \% / 50 \%$, and thoracotomy versus sternotomy was 9 versus 21 . All procedures were done successfully, but one patient had a periprocedural valve-in-valve implantation. Twenty-two patients were extubated in the operation room. The patients stayed in the intensive care unit for one night. Six patients were reoperated on. One patient had a postoperative balloon aortic valvuloplasty. The overall survival was $81 \%$ (follow up, 1-18 months).

Conclusions: Access design is an important issue in TAVI. When central approach is needed, TAVI-TAo is safe. For patients with low ejection fraction, the TAVI-TAo is preferred to the TAVI-transapical. The cannulation technique of the aorta is well known for cardiothoracic surgeons, and the method is feasible both for the Medtronic

Accepted for publication December 11, 2013

From the Department of Cardiothoracic Surgery, Oslo University Hospital, Oslo, Norway.

Presented at the Annual Scientific Meeting of the International Society for Minimally Invasive Cardiothoracic Surgery, June 12-15, 2013, Prague, Czech Republic.

Disclosures: Gry Dahle, MD, declares no conflict of interest. Kjell-Arne Rein, $\mathrm{MD}, \mathrm{PhD}$, is a proctor for Edwards Lifesciences, Irvine, CA USA.

Address correspondence and reprint requests to Gry Dahle, MD, Department of Cardiothoracic Surgery, Oslo University Hospital, Rikshospitalet, Postboks 4950, Nydalen 0424 Oslo, Norway. E-mail: gdahle@ous-hf.no; g_dahle@hotmail.com.

Copyright (C) 2014 by the International Society for Minimally Invasive Cardiothoracic Surgery

ISSN: 1556-9845/14/0901-0001
CoreValve and the Edwards SAPIEN valve, either via right minithoracotomy or ministernotomy to obtain the best coaxial alignment. It seems easier to position the bigger valves more precisely via this central approach.

Key Words: TAVR, Transcatheter valve replacement, Direct aorta, Ministernotomy, Minithoracotomy, Edwards SAPIEN, CoreValve.

(Innovations 2014;9:1-9)

Transcatheter aortic valve implantation (TAVI) is currently used to treat high-risk and inoperable patients with aortic stenosis. ${ }^{1}$ The established access routes are either retrograde transfemoral (TF) and/or antegrade transapical (TA) $)^{2,3}$; more recently, approaches via the subclavian artery ${ }^{4}$ or via the carotid artery have also been used. The TA access is not always feasible and is sometimes associated with myocardial damage as well as bleeding, postprocedural chest pain, and pleural effusion. The transaortic approach (TAo) has therefore emerged as an alternative to central access. ${ }^{5,6}$ This direct aorta ascending approach can be performed by either a mini J-shaped upper sternotomy or a right minithoracotomy in the second or the third intercostal space. Both the Edwards SAPIEN (Edwards Lifesciences, Irvine, CA USA) and CoreValve (Medtronic, Minneapolis, MN USA) systems are suitable for the TAo approach (Fig. 1). The CoreValve got the CE (Conformité Européenne) marking for TAVI-TAo in November 2011 and the SAPIEN valve half a year later.

After an initial experience with TA, the TAo has nowadays become the first choice as the central access route in our institution. The TA is more often used for intracardiac, leftsided (ie, mitral and left atrium) procedures. We describe our TAo technique and the early experience with 30 patients.

\section{METHODS}

\section{Patient Selection}

The patients are reviewed by a "heart team" of surgeons and cardiologists after being judged inoperable or at high risk for surgical aortic valve replacement (AVR). Multislice computed tomography (CT) and transesophageal echocardiography (TEE) in addition to coronary angiography and transthoracic echocardiography are performed for each patient. Central access is preferred if the femoral arteries are very tortuous, have a diameter that is too small, or have circumferential calcification. The central access is used if the patient has an abdominal or an iliac aneurysm, and we also consider central access if the 


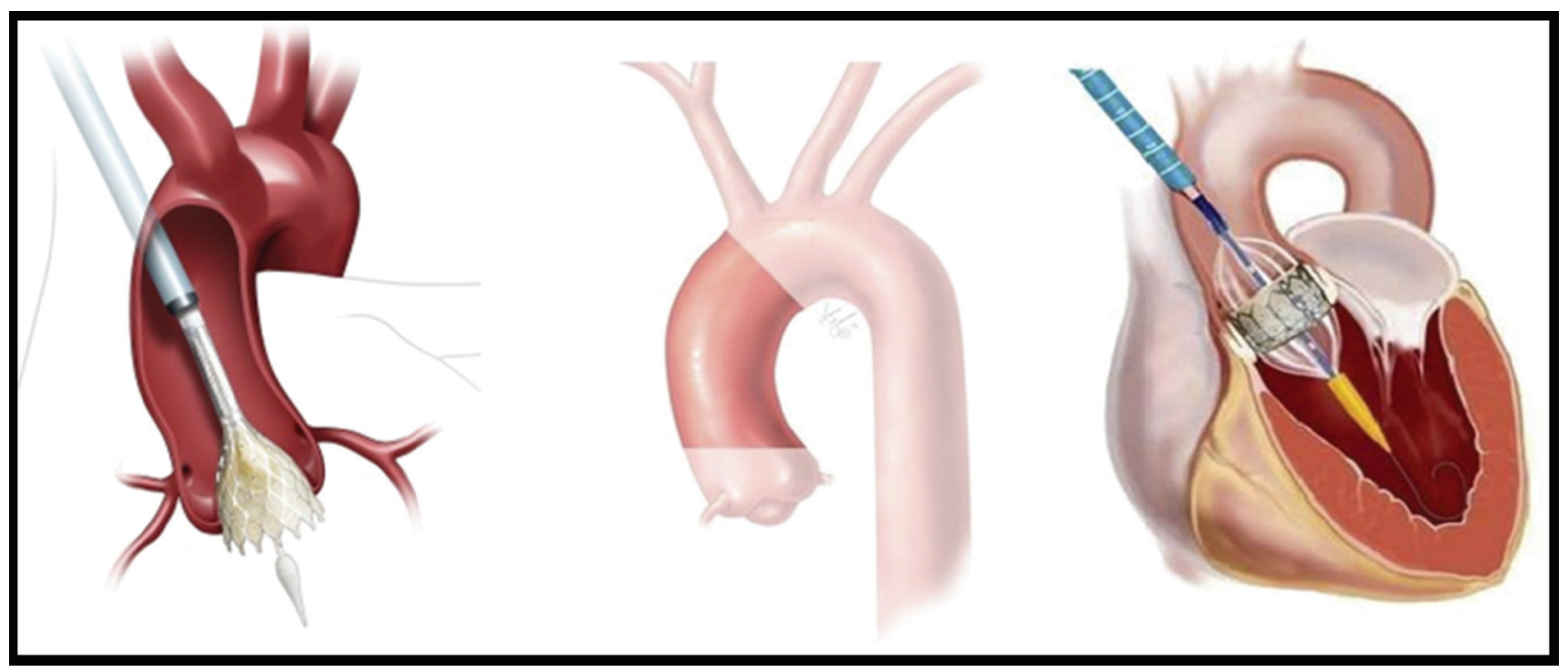

FIGURE 1. The two THV systems for transaortic TAVI. The CoreValve to the left and the Edwards SAPIEN-XT to the right. In the middle, the aorta ascendens with the target zone for the introducer. TAVI indicates transcatheter aortic valve implantation; THV, transcatheter heart valve.

patient is very obese. We then do the angiography from the radial artery.

The configuration of the ascending aorta is evaluated on the CT scan. To have the best coaxial alignment from the puncture site on the aorta to the annular plane, either a sternotomy or a thoracotomy is chosen. If previous coronary artery bypass graft $(\mathrm{CABG})$ operation has been performed, the position of the left internal mammary artery (IMA)/right IMA and vein grafts is considered. The length of the ascending aorta is measured, and any plaques of calcification are evaluated for the sheath insertion. Mini J-sternotomy is preferred when the ascending aorta is in the midline or toward the left side, more than $6 \mathrm{~cm}$ deep to the sternum, and the IMA/vein grafts are not attached to the posterior part of the sternum (Fig. 2). Right minithoracotomy is preferred when the ascending aorta is more than $50 \%$ on the right side of the midline, having a very horizontal extension, and is less than $6 \mathrm{~cm}$ deep to the chest wall ${ }^{7}$ (Figs. 3A, B).

The sizing of the transcatheter heart valve (THV) is done by measuring the perimeter, the surface area, or the length of the shortest and longest diagonal on the CT reconstruction in the OsiriX (Pixmeo, Geneva, Switzerland) software program.

According to valve size, shape of the annulus, need of pacing, and length of the aorta ascendens, the most appropriate valve is selected. CoreValve is preferred when the ventricular
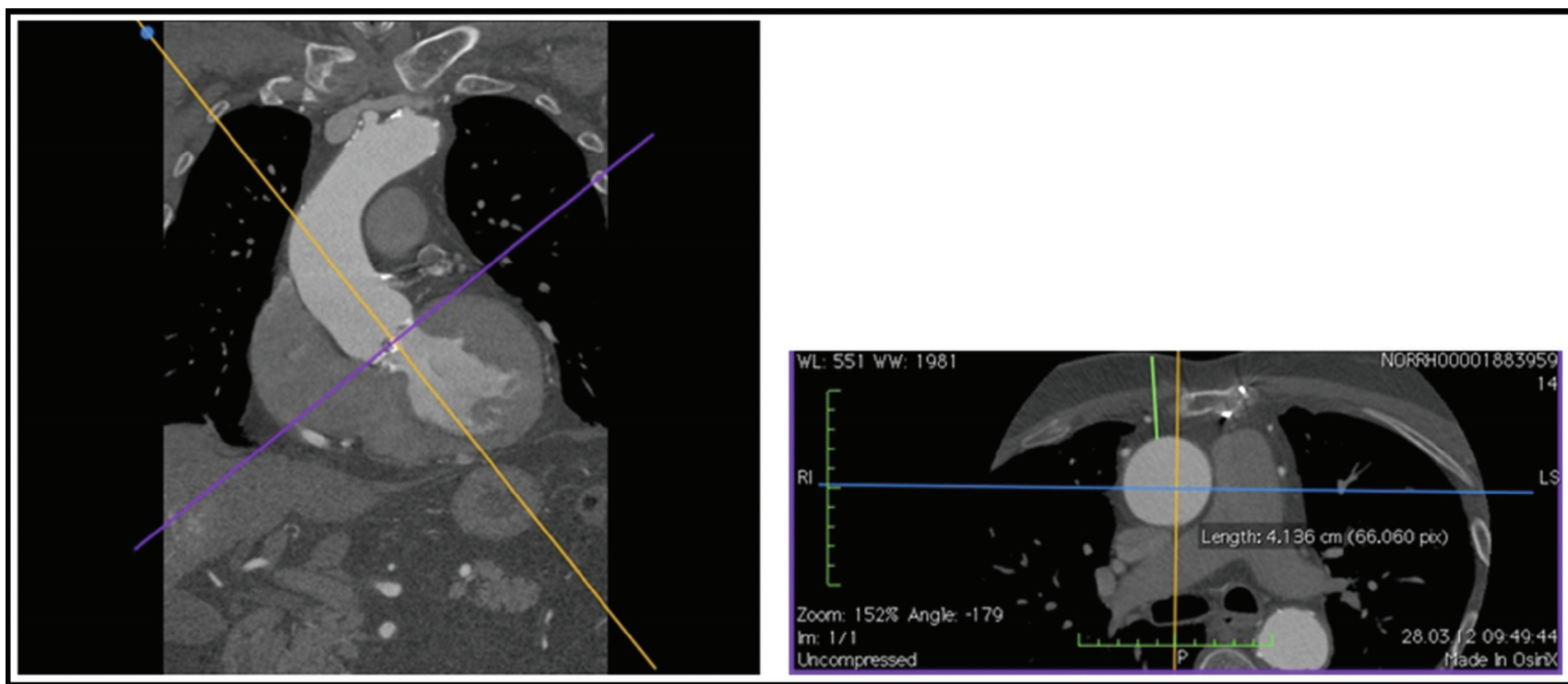

FIGURE 2. Ministernotomy preferred: vertical aorta on the CT reconstruction. The aorta is located centrally in the mediastinum. 


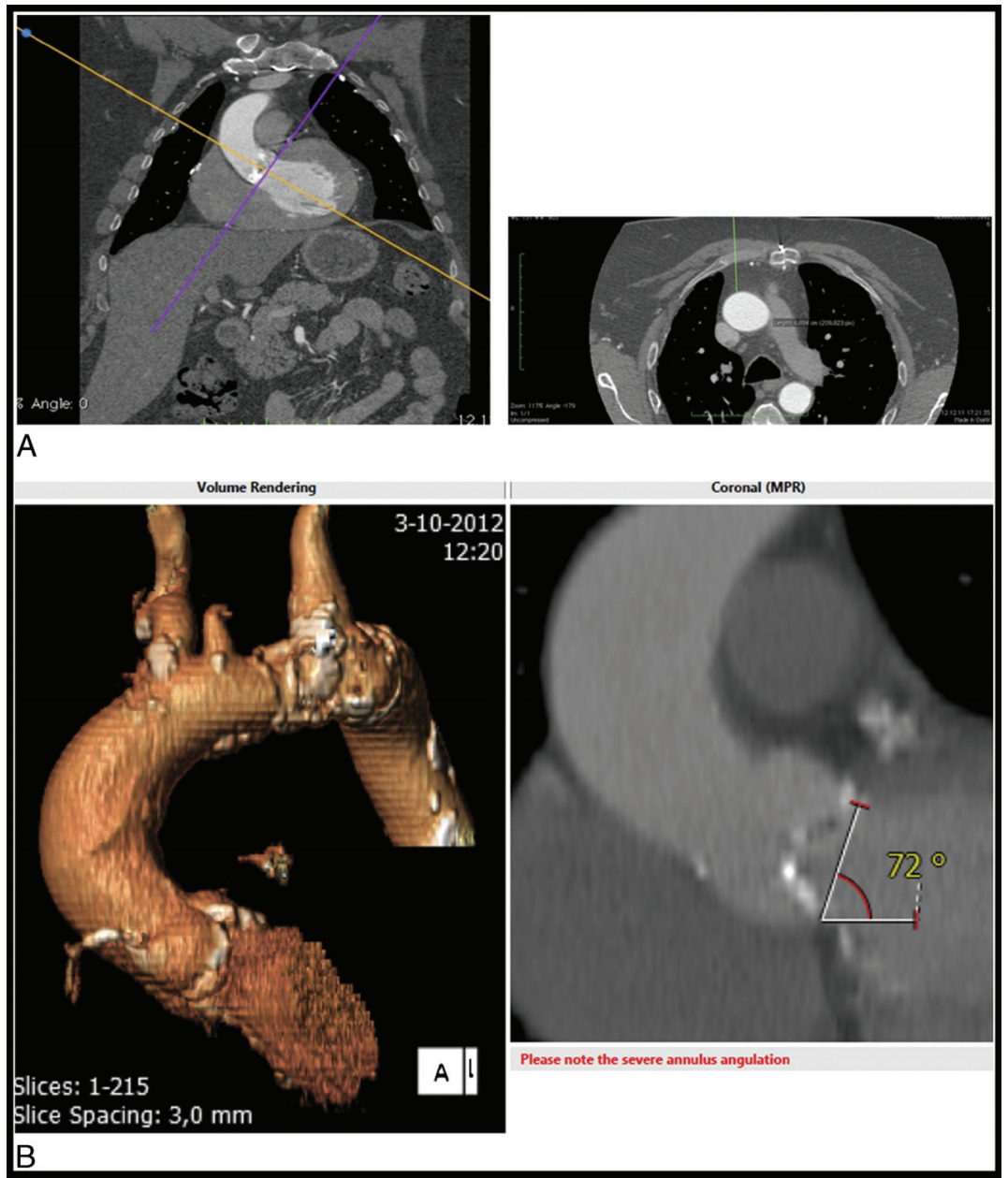

FIGURE 3. A, Right minithoracotomy preferred: horizontal aorta on the CT reconstruction. The aorta is situated mainly to the right side in the mediastinum. B, A horizontal aorta. Notice the angle of 72 degrees to the aortic annulus. MPR indicates multiplanar reconstruction.

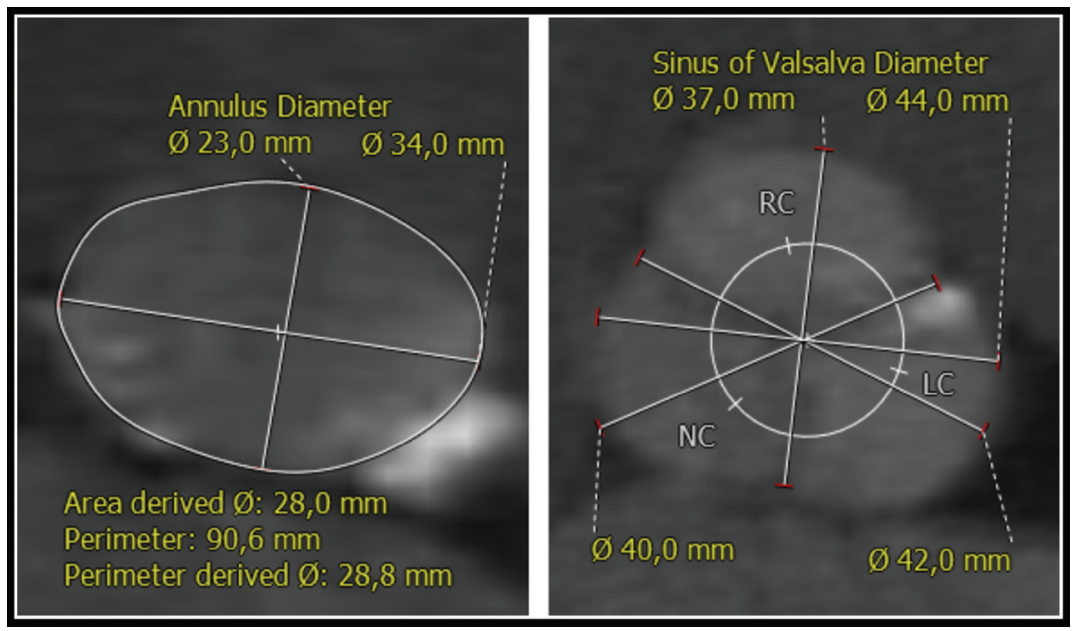

FIGURE 4. Annulus sizing. An oval annulus. Sizing by area, perimeter, or mean diameter on the CT scan to have the correct size of the catheter valve to be used. 


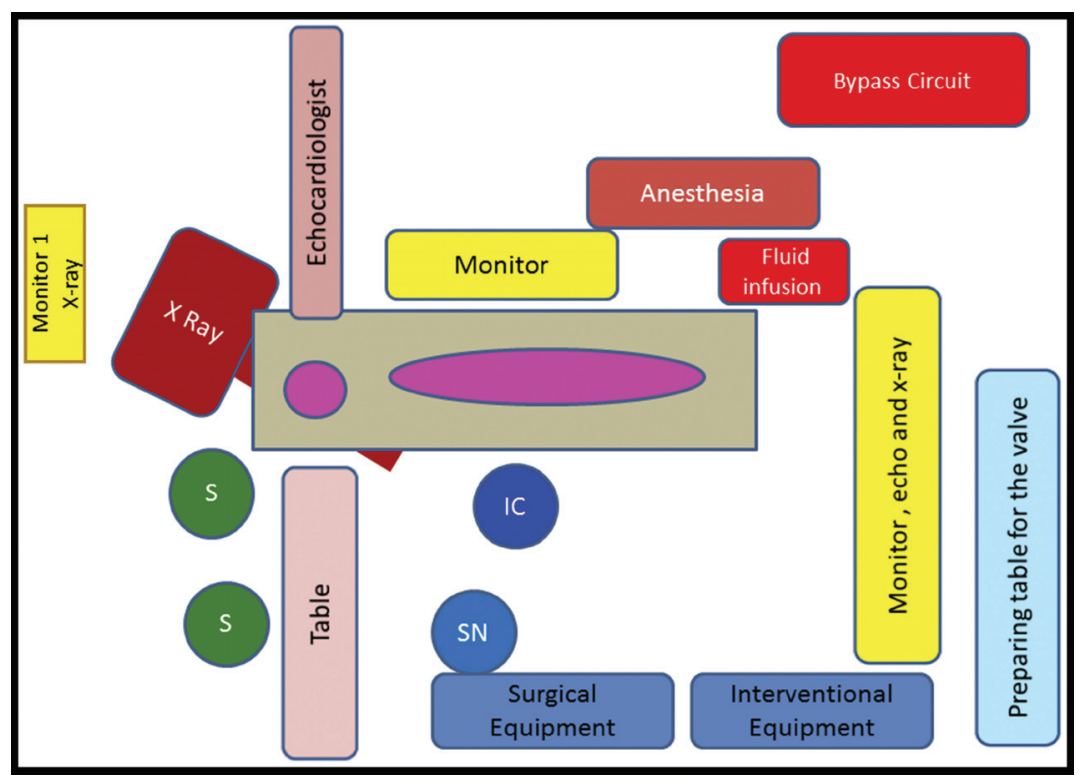

FIGURE 5. Room setup. S: operating cardiothoracic surgeon. IC: interventional cardiologist. SN: scrub nurse. The surgeons are positioned at the head of the patient, the interventional cardiologist alongside the angiography table, and the anesthesiologist is situated at the feet of the patient (modified with permission from Vinyac Bapat Guys and St. Thomas' Hospital, London, UK, and Leo Ihlberg, Helsinki University Hospital, Helsinki, Finland).

function is reduced to minimize the need of rapid pacing as well as for the oval-shaped and bigger annuli sizes (31 mm) (Fig. 4). Otherwise, the valves are equally chosen.

\section{Operative Procedure}

All operations are done in our hybrid operating room under TEE, fluoroscopy, and angiography guidance. A perfusionist and a heart-lung machine ready for use are present in the room. The patient is usually in general anesthesia owing to the discomfort with the TEE probe.
The room "setup" when doing TAo differs somewhat from that of the TA and TF approaches. The two THV operators are positioned at the head of the patient, whereas the anesthesiologist is situated at the foot of the operating table (Fig. 5).

A pigtail catheter is positioned into the aortic root, usually from a femoral puncture. A transvenous temporary pacing wire for rapid ventricular pacing during the procedure is introduced either through the femoral or the internal jugular vein. Two extra 5F and 6F multipurpose sheaths are put into the femoral artery and vein as a "safety net" if a femoral-femoral bypass is needed.

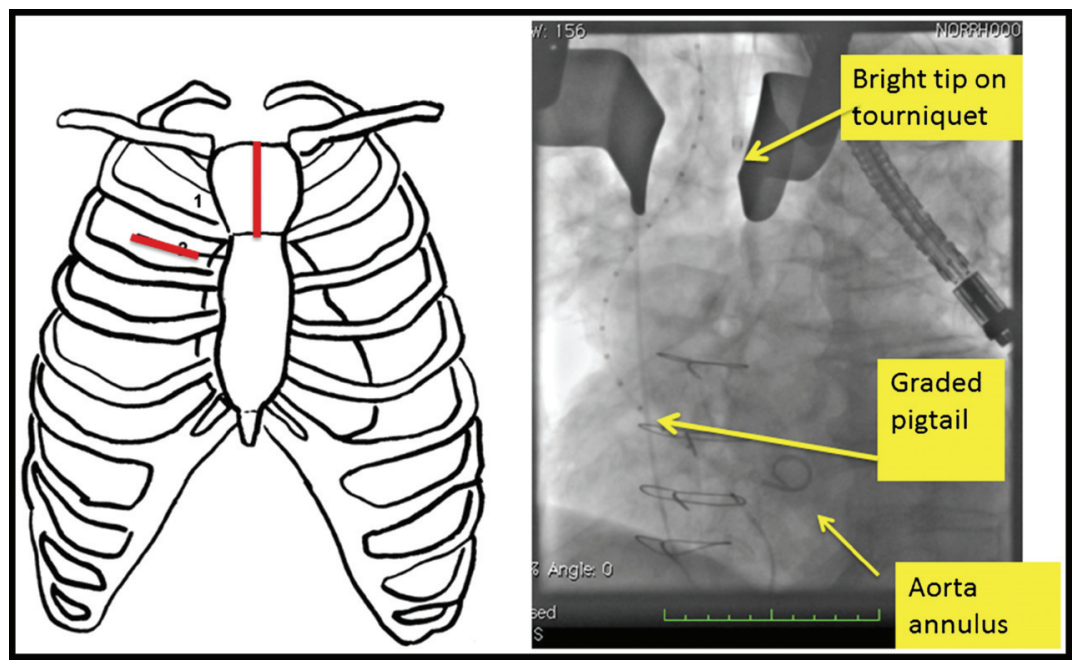

FIGURE 6. The operation. Lines on the figure illustrate the ministernotomy and the minithoracotomy. A radiopaque tourniquet tip on the purse-string suture at the level of the puncture site on the ascending aorta. A graded pigtail catheter is used to measure distance to the aortic annulus. 


\begin{tabular}{lc}
\hline TABLE 1. Demographics & \\
\hline & Patients $(\mathbf{n}=\mathbf{3 0})$ \\
\hline Age, y & $80(67-89)$ \\
Male sex & 18 \\
BSA, kg/m ${ }^{2}$ & $1.83(1.53-2.62)$ \\
Diabetes & 9 \\
COPD & 10 \\
CAD & 18 \\
Redo surgery & 16 \\
Pacemaker implanted & 3 \\
Atrial fibrillation & 10 \\
NYHA class III/IV & 30 \\
AAA & 6 \\
Log EuroSCORE & $33(15-67)$ \\
STS score mortality & $6.59(2.54-16.55)$ \\
STS score morbidity & $28.44(18.60-50.34)$ \\
\hline
\end{tabular}

AAA indicates abdominal aortic aneurysm; BSA, body surface area; CAD, coronary artery disease; COPD, chronic obstructive pulmonary disease; NYHA, New York Heart Association; STS, The Society of Thoracic Surgeons.

\section{Mini J-sternotomy}

A midline incision of 5 to $6 \mathrm{~cm}$ from the jugulum to the level of the third rib is performed. A small oscillating sternal saw is used to do the sternotomy extended into the second or the third intercostal space on the right side. A mini sternal retractor is applied. The pericardium is kept away by stay sutures to expose the ascending aorta.

2. Right minithoracotomy

A 4-cm-long skin incision is performed from the right sternal border in the second intercostal space. The pleura are entered and the right IMA is usually ligated. Exposure of the ascending aorta is achieved using a soft tissue retractor and/or a small rib spreader. The pericardium is identified and divided parallel to the skin incision and retracted with pericaridal stay sutures.

3. Combined ministernotomy and right thoracotomy

A ministernotomy extended to the right side by a minithoracotomy is an option in redo situation.

\section{Purse-String Sutures on Ascending Aorta}

The site of the purse-string sutures is decided by fluoroscopy at least 6 to $7 \mathrm{~cm}$ above the annulus level. A pigtail catheter with 1-cm-spaced radiopaque markers is used to measure this distance. A noncalcified spot on the anterior aspect of the ascendens is determined by palpation. Two purse-string sutures of polypropylene 3-0 are then made, the inner circle without pledgets and the outer one with 4-5 Teflon pledgets (PTFE polymer pledget, Covidien, Mansfield, MA USA) of $3 \times 7 \mathrm{~mm}$. The sutures are secured by snares, and the width of the circle was big enough for the introducer sheath to be used (18F or 24F) (Fig. 6).

\section{Stiff Guide Wire Positioning and Balloon Aortic Valvuloplasty}

Heparin is given to provide activated clotting time of more than 250 seconds. The aorta is punctured with a needle, and a short guide wire is inserted. The needle is replaced by a 6F multipurpose sheath. With the help of an Amplatz left AL1 (Boston Scientific, Natick, MA USA) guiding catheter and a 0.035 -in straight-tipped wire, the aortic valve is crossed. Finally, an Amplatz extrastiff guide wire with a 1-cm soft tip preshaped like a pigtail is carefully positioned into the left ventricle under fluoroscopy.

The Ascendra+ sheath 24F/26F (Edwards Lifesciences, Irvine, CA USA) for the Edwards SAPIEN valve and the Cook -cm Check-Flo Performer 18F introducer sheath (William Cook Europe, Bjaerverscov, Denmark) for the Medtronic CoreValve are introduced into the ascending aorta and kept $2 \mathrm{~cm}$ into the lumen of the vessel. Balloon aortic valvuloplasty is performed under rapid pacing with the most appropriate balloon. ${ }^{7,8}$ Care is taken while removing the balloon so the extrastiff wire remains in place.

\section{Edwards SAPIEN Valve Implantation}

The SAPIEN-XT valve is crimped either on the Ascendra + system having a nose cone or on the former Ascendra II delivery system without any nose cone, described by Bapat and Attia. ${ }^{7}$ The delivery device is clicked onto the sheath and deaired, and the SAPIEN valve is gently pushed forward to cross the native valve. The pusher is pulled back, and the final adjustment is done to deploy the SAPIEN-XT valve as perpendicular as possible to the annular plane, approximately $50 \%$ above and $50 \%$ below the aortic annulus. Under apnea, rapid pacing (180 beats per minute), and an aortography being performed simultaneously, the valve is implanted slowly, allowing for a controlled repositioning during balloon expansion.

\section{Medtronic CoreValve Implantation}

The CoreValve is loaded on the delivery catheter and carefully advanced through the Cook sheath and across the native aortic valve. The release of the valve is performed stepwise by the use of repeated doses of contrast injection. Fast pacing is often used to maintain a satisfactory blood pressure during the most critical part of the deployment. Adjustment and repositioning are performed during the first two thirds of the release. ${ }^{8}$

In both circumstances, the short distance from the aortic puncture to the annulus allows a very precise valve implantation. Assessment of valve function, valve position, and possible paravalvular leak as well as coronary artery flow is made with TEE and angiography. The stiff guide wire and delivery system are withdrawn. The sheath is removed, the purse-string sutures are tied, and BioGlue was used (CryoLife, Europa Ltd, Hampshire, UK). Protamine is eventually given to neutralize some of the effects of heparin.

\section{Closure}

1. Mini J-sternotomy: the pericardium is left open and a $28 \mathrm{~F}$ chest drain or a Blake drain is used. The sternum is closed

\section{TABLE 2. Echocardiography Findings}

\begin{tabular}{lc}
\hline Valve area, $\mathrm{cm}^{2}$ & $0.7(0.4-0.8)$ \\
Aortic gradient, $\mathrm{mm} \mathrm{Hg}$ & $50(30-83)$ \\
Ejection fraction, \% & $41(25-50)$ \\
LVOT measured in TEE, mm & $23(18-28)$ \\
\hline
\end{tabular}

Values are presented as mean (range).

LVOT indicates left ventricular outflow tract; TEE, transesophageal echocardiography. 
with two to three sternal wires. The incision of 4 to $7 \mathrm{~cm}$ is closed in layers, and local anesthesia was provided (Fig. 5).

2. Right minithoracotomy: the pericardium is left open and a $28 \mathrm{~F}$ chest tube was applied to drain the right pleural cavity. The intercostal space is closed by one or two Vicryl 0-1 sutures (Ethibond, Johnson \& Johnson, New Brunswick, NJ USA). Incision is closed in layers, and local anesthesia was provided.

\section{Anticoagulation/Antiplatelet Therapy and Postprocedural Care}

The patients are usually extubated in the operating room if possible. All of them are monitored in the intensive care unit (ICU) for at least 24 hours. Both aspirin and clopridogel are given in the evening on the operating day. Because epicardial wires cannot be placed, the temporary transvenous pacemaker wires are kept for 24 hours for the Edwards SAPIEN valve and removed if there are no conduction abnormalities. The pacemaker wires are maintained for 5 to 7 days for the CoreValve patients and then removed if no atrioventricular block occurs. As long as the patients keep the transvenous pacemaker leads, they are on antibiotic prophylaxis.

\section{MATERIALS}

From September 2009 to May 2013, a total of 131 patients underwent THV therapy in our institution with either the Edwards SAPIEN-XT valve or the Medtronic CoreValve system, and of these, 30 patients had a TAo.

Baseline characteristics are shown in Table 1. There were 18 male patients, and the mean age was 80 (range, 67-89) years. All patients were in New York Heart Association functional class III to IV, the mean Logistic EuroSCORE was 33 (range, 15-67), and the mean STS (The Society of Thoracic Surgeons) mortality score was 6.6 (range, 2.5-16.6). Sixteen patients had previously undergone open heart surgery $(\mathrm{CABG})$, three patients had a pacemaker implanted, and six patients had abdominal aortic aneurysm (AAA). All patients had severe aortic stenosis with a mean pressure gradient of $50 \mathrm{~mm} \mathrm{Hg}$ and mean opening area of $0.7 \mathrm{~cm}^{2}$ (Table 2).

An equal number of SAPIEN-XT and CoreValve were implanted, and more often, the biggest available valve sizes were used. The access route through a mini J-sternotomy was the most frequent one (Table 3 ).

\section{RESULTS}

The procedural success was $97 \%$; one patient underwent a periprocedural valve-in-valve implantation because of an initial high deployment of the first valve. Twenty-two patients were extubated in the operating room, and the mean ICU stay was one night.

There were no cerebrovascular events, coronary obstruction, or annulus rupture. grade 1.

Twenty-four patients had paravalvular leak of less than

Five patients had pacemaker implantation after the procedure, three patients had CoreValve 31-mm prosthesis, and two patients had Edwards SAPIEN-XT 29-mm prosthesis.

\begin{tabular}{|c|c|}
\hline & $\mathbf{N}=\mathbf{3 0}$ \\
\hline Thoracotomy/sternotomy/combined & $7 / 21 / 2$ \\
\hline $\mathrm{ES} / \mathrm{CV}$ & $15 / 15$ \\
\hline ES 23 & 1 \\
\hline ES 26 & 7 \\
\hline ES 29 & 7 \\
\hline CV 23 & 0 \\
\hline CV 26 & 3 \\
\hline CV 29 & 3 \\
\hline CV 31 & 9 \\
\hline Valve size, mean, mm & 28 \\
\hline Coronary obstruction, $\mathrm{n}$ & 0 \\
\hline Annulus rupture & 0 \\
\hline Aortic dissection & $0^{*}$ \\
\hline Procedure on bypass & $0 \dagger$ \\
\hline Valve-in-valve perioperative & 1 \\
\hline Procedural success, $\%$ & 97 \\
\hline Procedural time, mean (range), min & $137(93-466)$ \\
\hline Operating room time, mean (range), min & $250(114-551)$ \\
\hline
\end{tabular}

There was a huge difference of approximately 107 minutes in "procedural time" (mean, 136 minutes) and "operating room time" (mean, 243 minutes).

The 30-day survival rate was $93 \%$, and the overall survival was $87 \%$ (observation time, 1-18 months) (Tables 3, 4).

According to the VARC (Valve Academic Research Consortium) criteria on standardized endpoint definitions in TAVI, ${ }^{9,10}$ there were some adverse events and some minor clinical complications.

In one patient, there was a left ventricular perforation of the stiff guide wire just after valve deployment. Open surgery and a successful repair of the perforation were completed on partial bypass. A second patient had a pacemaker wire perforation of the right ventricle in the ICU a few hours after the TAVI procedure and underwent an urgent reoperation. In addition, one patient got a very low implantation of the catheter valve, and in the follow-up, he developed a severe paravalvular leakage (PVL) and was readmitted for open redo surgery (Fig. 7) some months later. A surgical valve was then successfully implanted.

Minor adverse events occurred such as hemothorax in two patients; one needed a chest drain postoperatively and another one underwent a local wound reintervention to stop the bleeding. One patient had a femoral artery reconstruction because of failure of the preclose device after removal of the angiocatheter. Intra-aortic balloon pump was used for 1 day after TAVI in one patient because of reduced left ventricular function (ejection fraction, $<17 \%$ ).

One patient had a postdilatation of the valve 2 days after the TAVI procedure. The valve had not been fully expanded, creating a severe PVL initially not verified during the valve implantation. A femoral access was chosen for the balloon aortic valvuloplasty and sealed off completely the PVL. However, a short dissection of the ascending aorta occurred 


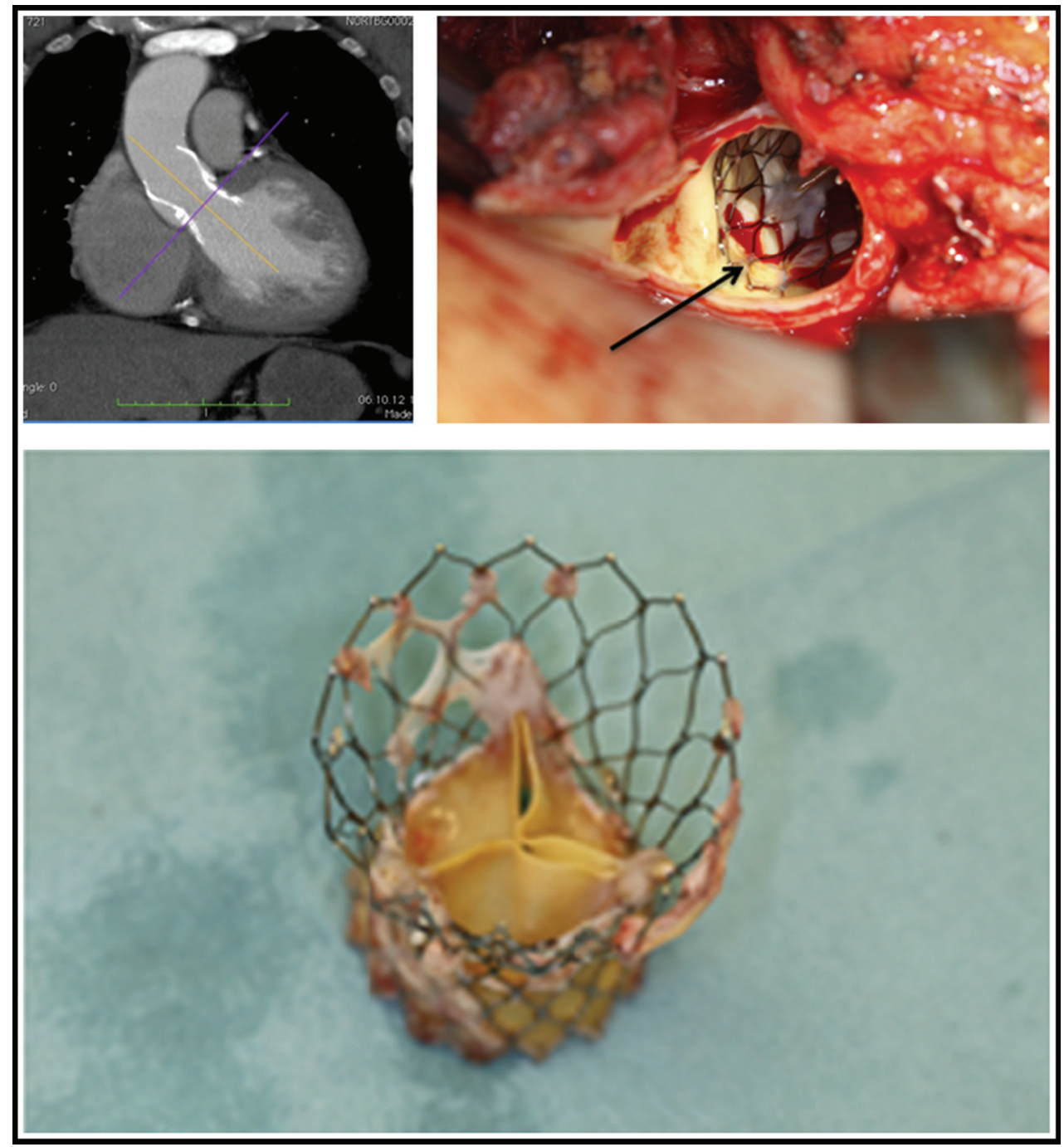

FIGURE 7. Redo surgery. Low position of the CoreValve after transaortic TAVI with paravalvular leak. Open redo surgery is performed. Following the aortotomy, the arrow is showing the area of paravalvular leak. The explanted CoreValve to the right. $\mathrm{TAVI}$ indicates transcatheter aortic valve implantation.

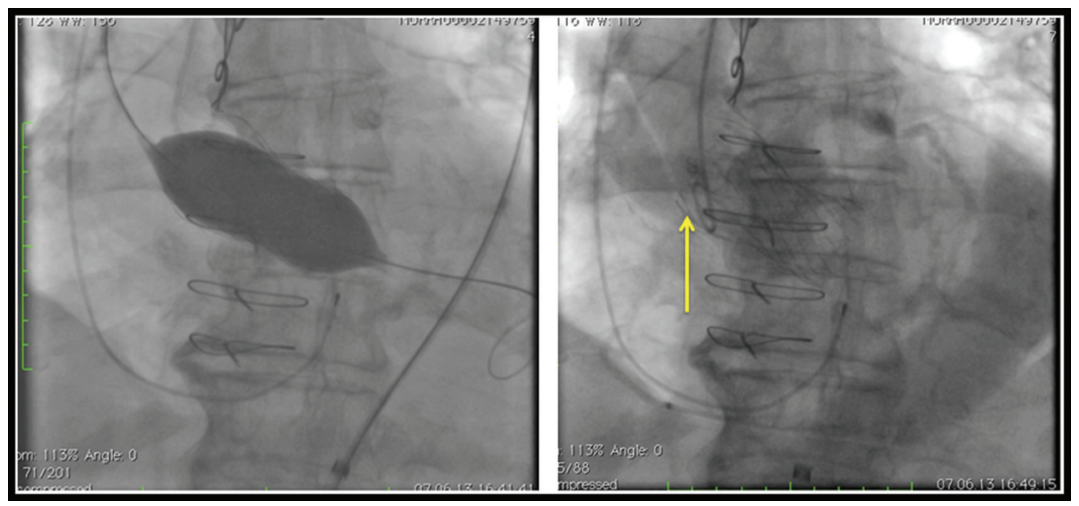

FIGURE 8. Postdilatation with femoral access and aortic dissection. After direct aortic procedure with CoreValve $31 \mathrm{~mm}$

the patient had a medium paravalvular leak, and a postdilatation was done successfully, eliminating the paravalvular leak. A local dissection was observed in the ascending aorta, conservatively treated. 


\begin{tabular}{lr}
\hline TABLE 4. Results & \\
\hline PVL $>$ 2, n & 6 \\
Extubated in operating room & 22 \\
IABP & 1 \\
Postoperative pacemaker implantation & 5 \\
Cerebrovascular events & 0 \\
30-d/total mortality & $2 / 4$ \\
\hline
\end{tabular}

IABP indicates intra-aortic balloon pump; PVL, paravalvular leak.

during this second procedure (Fig. 8), treated only conservatively (Table 5).

\section{DISCUSSION}

Transcatheter aortic valve implantation has become a well-established treatment option for patients who are regarded to be prohibitive or at high risk for conventional open aortic valve technique. Early results with TAVI using the SAPIEN and the CoreValve have shown encouraging results, with successful implantation in more than $90 \%$ of the procedures. ${ }^{1,11}$

Transapical TAVI is used in patients who are not suitable for TF implantation because of technical issues (small dimension and tortuousity of ileofemoral vessels as well as circular calcification of the ileofemoral trunk, AAA, and unfavorable aortic arch anatomy). The TA access is favorable because of its aspect of being antegrade through the aortic valve. Analysis of the TA patient demography has demonstrated that the population has a greater comorbidity and higher perioperative risk score than do TF patients. ${ }^{12-14}$ Hence, only in centers with great experience, using only the TA approach, the results are comparable with the TF results. ${ }^{15}$ However, there may be complications with the access including apical cannulation bleeding, late false aneurysm development, and difficulty in wire and valve positioning in patients with important septal hypertrophy as well as access difficulties in patients with left-sided chest problems such as previous thoracic pleurodesis or chest wall deformities. ${ }^{12,16,17}$ In our institution, the TA approach is preferentially used for intracardiac procedures, such as mitral valve interventions. Transapical approach may also be an option for transcatheter closure of PVL after surgical AVR and for left atrial appendage closure.

Transaortic TAVI has recently emerged in the literature and in clinical practice as a viable and safe technique for transcatheter valve implantation. ${ }^{5-7,18}$ For cardiac surgeons, there are many similarities compared with open heart surgery.
The approach through the ascending aorta is well recognized. With regard to the aorta purse-string closure, there is a wellknown precedent: "the safety of aortic puncture and repair is demonstrable in most open heart procedures."

The short distance from the puncture site to the aortic valve and the annulus facilitates the positioning because the movements of the catheter valve device are directly transmitted "one-to-one" and there is no delay in the system. In addition, with this access, it may be possible to "play with" the stiff wire for a precise positioning and alignment of the valve. If an intraprocedural problem occurs, the access route can easily be extended to a full sternotomy, allowing a regular open surgical approach to the heart.

Another aspect and a potential benefit of this new transaortic access route is the avoidance of entering the aortic arch and less manipulation of a calcified descending aorta. This may have an impact on the risk for stroke and the occurrence of neurological deficit. These statements are, however, yet to be proven.

The transaortic access route may also be combined with an open, conventional CABG in a hybrid procedure. The availability of fluoroscopy is then mandatory.

In this material, we observed no problem with the access route, although we experienced some adverse events such as ventricular perforation due to guide wire and pacemaker wire protrusion.

Two of our patients had to be converted to full sternotomy due to periprocedural complication (wire perforation/pacemaker wire protrusion). Probably, by using a less stiff guide wire when doing TAo, we may avoid the fear of ventricle perforation. The distance from the puncture site to the annulus is so short that the need of a stiff wire is not mandatory.

The third patient had a redo sternotomy and surgical AVR because of a significant PVL.

Hence, $10 \%$ of the patients with TAo approach had to undergo open sternotomy. This is similar to the other more conventional TAVI approaches such as TF and TA.

The difference between the "procedural" time and the "operation" time is mostly explained by the time consumption in the preoperative preparation as well as after the procedure because most of the patients are extubated in the operating room.

The patients for central access are often "multimorbid" with peripheral vessel disease and aneurysm formation. One patient in this registry had a stent graft because of an AAA

\begin{tabular}{lcccccc}
\hline \multicolumn{1}{l}{ TABLE 5. Adverse Events } \\
\hline Patient & M/F & Age, $\mathbf{y}$ & CV/ES & Size, $\mathbf{~ m m ~}$ & TH/ST & Description \\
\hline 8 & M & 72 & CV & 31 & TH & Low implantation of the valve, PVL, heart failure, open surgery \\
9 & M & 82 & ES & 29 & TH & Chest drain, hemothorax \\
11 & M & 72 & ES & 29 & ST & Preclose failure after angiography, femoral reconstruction \\
13 & F & 70 & CV & 26 & ST & Pacemaker perforation in ICU, acute surgery \\
14 & F & 78 & CV & 29 & TH & High implantation, valve-in-valve periprocedural \\
17 & F & 77 & ES & 26 & TH & Wire perforation after implantation, open repair of the left ventricle \\
20 & F & 83 & ES & 26 & Hemothorax, surgical pleura revision \\
28 & M & 78 & CV & 31 & Postdilation due to PVL 2 d after implantation \\
\hline
\end{tabular}

CV indicates CoreValve; ES, Edwards SAPIEN-XT; F, female; ICU, intensive care unit; M, male; PVL, paravalvular leakage; ST, sternotomy; TH, thoracotomy. 
after the TAVI procedure, and another patient is still waiting for an endovascular aneurysm repair treatment.

One patient had a postdilation (balloon aortic valvuloplasty) from a femoral access. This was a very difficult procedure because of heavy calcification of the ileofemoral trunk and proved that our initial decision for central access was correct. None of our patients had any cerebral events; probably, the avoidance of wires through the aortic arch can be an explanation.

\section{CONCLUSIONS}

Access design is an important issue in TAVI. When central approach is needed, transaortic TAVI is safe. Compared with the TA, the direct aorta access is the preferred method when there is a low ejection fraction and fragile ventricle. The delivery system is easily guided into the ascending aorta, with a short distance to the native valve and the annulus, thereby avoiding the long retrograde passage in a severely diseased aorta. The cannulation technique of the aorta is well known for cardiothoracic surgeons, and this approach is feasible both for the Medtronic CoreValve system and the Edwards SAPIEN valve. The choice between either the right minithoracotomy or the mini J-sternotomy is decided to obtain the best coaxial alignment to the aortic annulus (for a horizontal aorta, preferentially, a right thoracotomy, whereas for a vertical aorta, often, a sternotomy). In addition, it seems easier to position a bigger catheter valve (CoreValve $31 \mathrm{~mm}$; SAPIEN-XT $29 \mathrm{~mm}$ ) more precisely via this central TAo.

\section{ACKNOWLEDGMENTS}

The authors thank Jo Eidet, who has given support with echocardiography and anesthesia during the procedures, and our colleagues in the team for the good collaboration: interventional cardiologists Lars Aaberge, Bjørn Bendz, and Anders Hervold; anesthesiologists Jan Fredirk Bugge, Jan Hovdenes, and Andreas Espinoza; and echocardiologists Jon Offstad and Jan Otto Beitnes, as well as the rest of the heart team at the Intervention Centre for great assistance and support.

\section{REFERENCES}

1. Vahanian A, Alfieri OR, Al-Attar N, et al. Transcatheter valve implantation for patients with aortic stenosis: a position statement from the European Association of Cardio-thoracic Surgery (EACTS) and the European Society of Cardiology (ESC), in collaboration with the European association of percutaneous cardiovascular interventions (EAPCI). Eur J Cardiothorac Surg. 2008;34:1-8.

2. Walther T, Möllmann H, van Linden A, Kempfert J. Transcatheter aortic valve implantation transapical: step by step. Semin Thorac Cardiovasc Surg. 2011;23:55-61.
3. Himbert D, Roy D, Brecker S, et al. Tools \& techniques: transcatheter aortic valve implantation: transfemoral approach. EuroIntervention. 2011; 6:784-785.

4. Caceres M, Braud R, Roselli EE. The axillary/subclavian artery access route for transcatheter aortic valve replacement: a systematic review of the literature. Ann Thorac Surg. 2012;93:1013-1018.

5. Bapat V, Thomas M, Hancock J, Wilson K. First successful trans-catheter aortic valve implantation through ascending aorta using Edwards SAPIEN THV system. Eur J Cardiothorac Surg. 2010;38:811-813.

6. Bapat V, Khawaja MZ, Attia R, et al. Transaortic transcatheter aortic valve implantation using Edwards Sapien valve: a novel approach. Catheter Cardiovasc Interv. 2012;79:733-740.

7. Bapat V, Attia R. Transaortic transcatheter aortic valve implantation: stepby-step guide. Semin Thorac Cardiovasc Surg. 2012;24:206-211.

8. Bruschi G, de Marco F, Botta L, et al. Direct aortic access for transcatheter self-expanding aortic bioprosthetic valves implantation. Ann Thorac Surg. 2012;94:497-503.

9. Leon MB, Piazza N, Nikolsky E, et al. Standardized endpoint definitions for transcatheter aortic valve implantation clinical trials: a consensus report from the Valve Academic Research Consortium. J Am Coll Cardiol. 2011;57:253-269.

10. Kappetin AP, Head S J, Généreux P, et al. Updated standardized endpoint definitions for transcatheter aortic valve implantation: the Valve Academic Research Consortium-2 consensus document. J Thorac Cardiovasc Surg. 2013;145:6-23.

11. Piazza N, Grube E, Gerckens U, et al. Procedural and 30-day outcomes following transcatheter aortic valve implantation using the third generation (18 Fr) corevalve revalving system: results from the multicentre, expanded evaluation registry 1-year following CE mark approval. EuroIntervention. 2008;4:242-249.

12. Thomas M, Schymik G, Walther T, et al. Thirty-day results of the SAPIEN aortic Bioprosthesis European Outcome (SOURCE) Registry: a European registry of transcatheter aortic valve implantation using the Edwards SAPIEN valve. Circulation. 2010;122:62-69.

13. Dworakowski R, MacCarthy PA, Monaghan M, et al. Transcatheter aortic valve implantation for severe aortic stenosis - a new paradigm for multidisciplinary intervention: a prospective cohort study. Am Heart J. 2010;160:237-243.

14. Dewey TM, Brown DL, Herbert MA, et al. Effect of concomitant coronary artery disease on procedural and late outcomes of transcatheter aortic valve implantation. Ann Thorac Surg. 2010;89:758-767.

15. Pasic M, Buz S, Dreysse S, et al. Transapical aortic valve implantation in 194 patients: problems, complications, and solutions. Ann Thorac Surg. 2010;90:1463-1469.

16. Cribier A, Eltchaninoff H, Tron C, et al. Early experience with percutaneous transcatheter implantation of heart valve prosthesis for the treatment of end-stage inoperable patients with calcific aortic stenosis. $\mathrm{J} \mathrm{Am} \mathrm{Coll}$ Cardiol. 2004;43:698-703.

17. Cribier A, Eltchaninoff H, Bash A, et al. Percutaneous transcatheter implantation of an aortic valve prosthesis for calcific aortic stenosis: first human case description. Circulation. 2002;106:3006-3008.

18. Bruschi G, De Marco F, Fratto P, et al. Alternative approaches for transcatheter self-expanding aortic bioprosthetic valves implantation: singlecenter experience. Eur J Cardiothorac Surg. 2011;39:e151-e158.

\section{CLINICAL PERSPECTIVE}

This is a clinical report from Drs Dahle and Rein in Oslo, Norway, examining a series of 30 transaortic valve implantations. Nine patients had a right minithoracotomy; and 21, a ministernotomy incision. The procedural success rate was $97 \%$; the 30 -day survival rate was $93 \%$, with an overall survival rate of $87 \%$. The postoperative complication rate was low.

This represents an excellent approach for transcatheter aortic valve replacement. The experience of this group and others would suggest that this has significant advantages over the transapical approach for patients who are not suitable for transfemoral implantation. The authors are to be congratulated on their outstanding results. 
